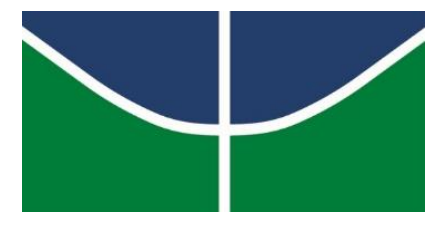

Universidade de Brasília

Instituto de Ciências Biológicas

Programa de Pós-Graduação em Ecologia

\title{
Partição de nicho isotópico por pequenos mamíferos em formações florestais de uma savana neotropical
}

\author{
Anna Carla Lima Camargo \\ Orientador: Prof. Dr. Emerson Monteiro Vieira
}

Dissertação apresentada junto ao Programa
de Pós-graduação em Ecologia como
requisito parcial para a obtenção do
título de Mestre em Ecologia.

Brasília, 25 de Abril de 2016 


\section{Agradecimentos}

Agradeço ao Departamento de Ecologia da Universidade de Brasília, ao Programa de Pósgraduação em Ecologia e aos professores do programa pelo auxílio durante todo o mestrado.

Ao Professor Dr. Emerson M. Vieira pela orientação e ajuda psicológica durante todo o processo. Imagino que não seja fácil ser psicólogo de tantos alunos com problemas o tempo todo.

À Professora Dra. Gabriela B. Nardoto pela orientação com os isótopos estáveis e pelas reuniões isotópicas que me ajudaram muito.

Às pessoas que me auxiliaram em campo (estagiários, técnicos, amigos e namorado), especialmente à Daniela Belts (hehehehe Behs), Nayara Sano e Thaiz Armond que estiveram sempre presentes como companheiras de campo e também grupo de apoio.

Aos colegas de laboratório, especialmente Nicholas Camargo, André Mendonça e Juliana Ribeiro, por me ajudarem com discussões a respeito dos meus dados, com análises e com o R.

Aos amigos e petas, principalmente Melissa Merlone, Guilherme Hirata e Heitor Campos, por aguentarem as longas ligações reclamando dos problemas de mestrado e sempre estarem dispostos a dividir uma batata do Outback.

Ao namorado, Vinícius Egler, que sempre esteve presente para me ouvir e me ajudar em tudo que eu precisasse. E também pela compreensão e paciência que você teve comigo nesse período.

Aos meus pais, Mila Lima e José R. Camargo pelo investimento para que eu pudesse chegar até essa fase e todo o carinho durante toda a minha vida.

À minha "rimã", Milla de Paula, que me apoiou e me ajudou a manter o ânimo.

Aos membros da banca pela disponibilidade em discutir e ajudar na conclusão dessa dissertação. Ao Amabílio de Camargo pelo auxílio na identificação dos artrópodes.

Ao Marcelo Kuhlmann pela identificação dos frutos.

À Coordenação de Aperfeiçoamento de Pessoal de Nível Superior (CAPES) pela bolsa de mestrado.

À equipe da Fazenda Água Limpa (FAL UnB), do Jardim Botânico de Brasília (JBB) e da Escola de Administração Fazendária (ESAF) pelo apoio e por me permitirem realizar a coleta de dados. 


\section{Sumário}

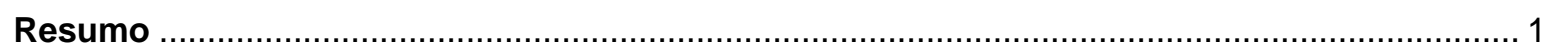

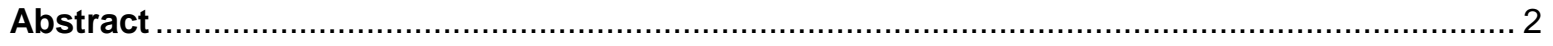

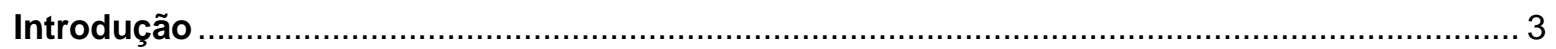

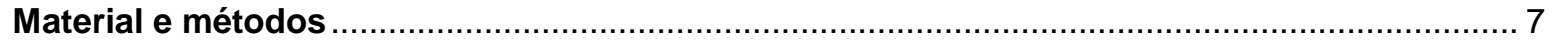

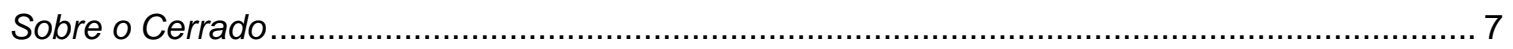

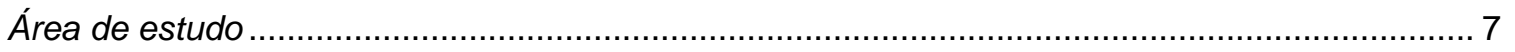

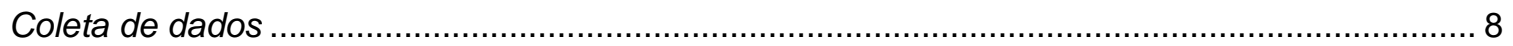

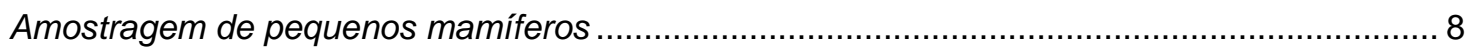

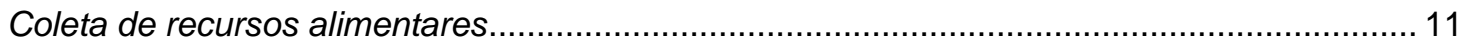

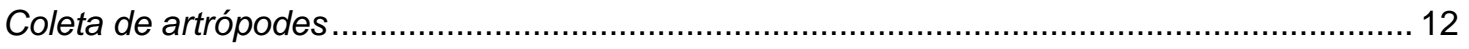

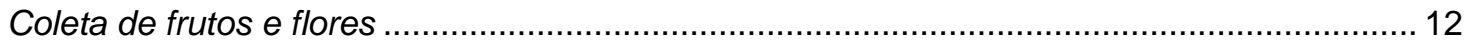

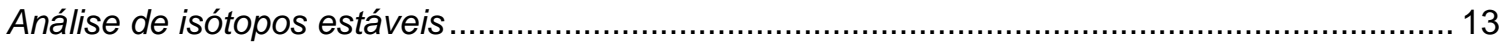

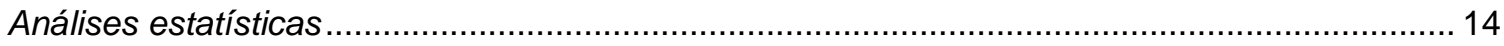

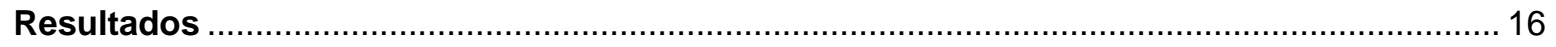

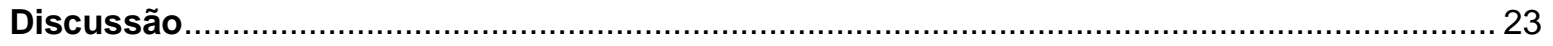

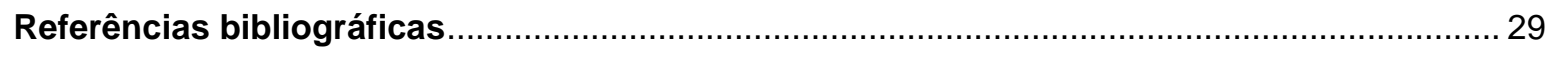

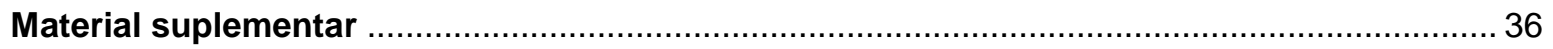




\section{Resumo}

A diferenciação das espécies quanto aos nichos ecológicos indica os mecanismos que possibilitam a coexistência de espécies similares. Pequenos mamíferos particionam recursos principalmente por meio da segregação nas dimensões alimentares e espaciais. Em florestas, a ocorrência de espécies relacionadas à complexidade estrutural pode resultar em mais nichos ecológicos. O Cerrado (savana neotropical) possui florestas estruturalmente distintas, como cerradão e mata de galeria. Essas características permitem a avaliação da partição de recursos por pequenos mamíferos entre fitofisionomias com comunidades similares, porém complexidades distintas. Usando isótopos estáveis de ${ }^{13} \mathrm{C}$ e ${ }^{15} \mathrm{~N}$, caracterizei a assimilação isotópica das espécies de pequenos mamíferos e comparei a partição de nicho isotópico entre conjuntos similares de espécies presentes nas duas fitofisionomias considerando que a mata de galeria é mais complexa estruturalmente. As espécies em ambas fitofisionomias demonstraram ser onívoras, diferenciando-se na proporção em que assimilam alimentos de origem vegetal e animal. Os marsupiais Didelphis albiventris, Gracilinanus agilis e Caluromys lanatus assimilaram mais artrópodes, enquanto que os roedores Rhipidomys macrurus, Hylaeamys megacephalus e Proechimys roberti assimilaram proporções similares de material vegetal e animal e os roedores Oecomys bicolor e Oligoryzomys nigripes assimilaram uma proporção maior de material vegetal com sinal de plantas C3 e C4. Roedores apresentaram maior amplitude de nicho isotópico em matas de galeria enquanto que marsupiais apresentaram menor amplitude de nicho nessas matas comparado com o cerradão. A comunidade de pequenos mamíferos de cerradão apresentou uma maior amplitude de $\delta^{15} \mathrm{~N}\left(\delta^{15} \mathrm{~N}\right.$ range), bem como maior amplitude geral e menor sobreposição de nicho isotópico em comparação com a comunidade da mata de galeria. Caluromys lanatus assimilou proporção maior de recursos de origem animal que o esperado, provavelmente devido ao consumo eventual de pequenos vertebrados. O sinal C4 das espécies $O$. bicolor e $O$. nigripes evidenciou o possível uso das áreas de campo próximas ou de clareiras nas matas de galeria por essas espécies. A menor amplitude total de nicho isotópico detectada na comunidade de pequenos mamíferos em mata de galeria contrariou minha expectativa inicial. Esse resultado pode ser devido às diferenças nas assinaturas isotópicas das plantas em cada tipo florestal. Apesar de ser menos complexo estruturalmente, o cerradão é mais heterogêneo e pode apresentar diversidade maior de plantas isotopicamente distintas que a mata de galeria. Esse padrão, por sua vez, é refletido na assinatura isotópica de invertebrados e de pequenos mamíferos. As maiores amplitudes de nicho dos roedores em mata de galeria comparada com àquelas apresentadas pelas mesmas espécies em cerradão refletem o consumo de uma diversidade maior de recursos isotopicamente distintos na mata de galeria. Isso indica que esse grupo apresenta maior plasticidade no uso de recursos alimentares que os marsupiais, e que a disponibilidade e diversidade de recursos no ambiente influencia a utilização de recursos e consequentemente a assimilação isotópica pelos pequenos mamíferos de florestas do Cerrado.

Palavras-chave: nicho, isótopos estáveis, marsupiais, roedores, cerrado 


\section{Abstract}

The differentiation of species regarding ecological niches indicates the mechanisms that allow the coexistence of similar species. Small mammals partition resources mostly through segregation on both food and spatial dimensions. In forests, the occurrence of species associated with structural complexity may result in more ecological niches. The Cerrado biome (neotropical savanna) possesses structurally distinct forests, such as cerradão (woodland forests) and gallery forests. These distinct characteristics allow comparisons of small-mammal resource partitioning between phytophysiognomies with similar communities but with distinct structural complexities. Using ${ }^{13} \mathrm{C}$ and ${ }^{15} \mathrm{~N}$ stable isotopes, I described isotopic assimilation of small-mammal species and compared the partitioning of isotopic niche between similar groups of species that occurred in both forest types, considering that gallery forests are structurally more complex than woodland forests. Species in both phytophysiognomies were omnivores, differing on the proportion at which they assimilate food resources from plant or animal origin. The marsupials Didelphis albiventris, Gracilinanus agilis, and Caluromys lanatus assimilated more arthropods, whereas the rodents Rhipidomys macrurus, Hylaeamys megacephalus, and Proechimys roberti assimilated similar proportions of plant and animal sources; and the rodents Oecomys bicolor and Oligoryzomys nigripes assimilated larger proportions of plant material with isotopic signals from both C3 and C4 plants. Rodents presented larger isotopic niche amplitude in gallery forests, whereas marsupials presented narrower niche amplitude in these forests compared to cerradão. The cerradão small mammal assemblage presented higher $\delta^{15} \mathrm{~N}$ range, increased general isotopic niche (TA) and decreased overlap in comparison to the gallery forest assemblage. Caluromys lanatus assimilated larger proportions of animal-origin resources than expected, probably due to eventual consumption of small vertebrates. The $\mathrm{C} 4$ isotopic signature of $O$. bicolor and $O$. nigripes species indicated a possible use of nearby grassland areas or forest gaps by these species. The smaller general isotopic niche width that I observed in the gallery forest small-mammal community contradicted my initial expectation. This result may be due to differences on plant isotopic signatures between forest formations. Although less structurally complex, the cerradão is more heterogeneous and may present higher diversity of isotopically distinct plants in comparison to gallery forest. This pattern, in turn, is reflected on invertebrates and small mammals' isotopic signatures. Increased rodents' isotopic niches in gallery forests compared to those presented by the same species in cerradão reflect the consumption of a larger diversity of isotopically distinct resources in gallery forest. This indicates a larger plasticity on the use of food resources by this group in comparison to the marsupials and also that both resource availability and diversity influence resource use and consequently isotopic assimilation by small mammals in Cerrado forests.

Keywords: niche, stable isotopes, marsupials, rodents, cerrado 


\section{Introdução}

Uma das questões centrais em ecologia é definir como as comunidades se estruturam e se organizam (Cody e Diamond 1975; Tilman 1982; Simberloff e Dayan 1991). Uma das formas operacionais de se entender como as comunidades se assemelham na sua essência de organização é por meio da avaliação do nicho ecológico das espécies que as compõem (Elton 1927). Nicho ecológico é um conceito que possui múltiplas definições (ver Grinnel 1917; Elton 1927; Hutchinson 1957). Segundo Hutchinson (1957), o nicho é um hipervolume composto por $n$ dimensões que descrevem o conjunto de condições e recursos que permitem o estabelecimento de uma espécie em determinada comunidade. Essas dimensões podem ser resumidas nos eixos bionomic e scenopoetic, referentes respectivamente aos recursos utilizados pelas espécies e ao intervalo de variáveis ambientais no qual a espécie melhor executa suas funções (Hutchinson 1978).

O padrão de organização de espécies ao longo das principais dimensões do nicho (e.g. dimensão espacial, alimentar ou temporal) reflete a estrutura da comunidade, bem como informa a respeito dos mecanismos que possibilitam a coexistência de espécies similares (Schoener 1974; Giller 1984). A importância relativa de cada dimensão varia de acordo com o táxon e com o hábitat avaliados, porém as espécies geralmente se diferenciam primeiramente no espaço, seguido da dimensão alimentar (ver revisão em Schoener 1974). Entretanto, para alguns táxons, mais de uma dimensão pode ser importante para se definir como as espécies particionam os recursos (Cody 1968; Rosenzweig e Winakur 1969; Pianka 1973; Meserve 1981).

Pequenos mamíferos particionam recursos principalmente por meio da segregação nas dimensões alimentares e espaciais (Brown e Lieberman 1973; Brown 1975; Emmons 1980; Meserve 1981; Bonaventura e Suárez 2001). Roedores de deserto norte-americano, por exemplo, particionam recursos com base tanto em tamanho de sementes quanto em área de forrageamento no plano horizontal (Brown e Lieberman 1973). Por outro lado, pequenos mamíferos em semi-árido chileno se diferenciam principalmente na dimensão alimentar no nicho e apresentam maior sobreposição na dimensão de hábitat (Meserve 1981). 
Os padrões de partição de recursos por pequenos mamíferos variam entre ecossistemas distintos. Diferentemente de desertos e sistemas semi-áridos, florestas apresentam um componente vertical da estrutura do habitat que pode proporcionar mais uma dimensão de nicho e abrigar uma maior diversidade de espécies relacionadas à complexidade do ambiente (Klopfer e MacArthur 1960; MacArthur e MacArthur 1961; August 1983; Grelle 2003; Vieira e Camargo 2012). Dessa forma, comunidades de pequenos mamíferos em ambientes complexos podem apresentar uma amplitude de nicho maior. A complexidade estrutural também pode permitir o empacotamento das espécies, ou seja, especialização no uso de recursos e a ocorrência de espécies morfologicamente similares em um mesmo local (MacArthur e Levins 1967; Grelle 2003)

A complexidade estrutural influencia o uso vertical do espaço por pequenos mamíferos que, por sua vez, pode explicar os padrões de partição de recursos alimentares e de dieta das espécies em diferentes formações florestais (Hannibal e Cáceres 2010; Leiner et al. 2010; Vieira e Camargo 2012). O maior número de estratos verticais permite que animais especializados em locomoção arbórea se aproveitem dos recursos alimentares presentes nas camadas superiores que estariam apenas eventualmente disponíveis para espécies terrícolas (Vieira e Camargo 2012). Além disso, a maior conexão entre os estratos verticais, possibilitada por lianas, facilita a locomoção tridimensional dos indivíduos durante o forrageamento (Hannibal e Cáceres 2010).

Florestas tropicais são de grande valia para estudos de partição de recursos alimentares por abrigarem uma alta diversidade de espécies que podem particionar recursos alimentares ao longo dos estratos verticais. Alguns estudos já investigaram o uso do espaço vertical e de recursos alimentares por pequenos mamíferos em sistemas florestais (Emmons 1980; Leite et al. 1996; Bonaventura e Suárez 2001; Sushma e Singh 2006; Symes et al. 2013). Entretanto, estes estudos são escassos nas florestas do bioma Cerrado (porém ver Hannibal e Cáceres 2010).

O Cerrado é um grande domínio vegetacional que contém cerca de 104 espécies de pequenos mamíferos não-voadores (ordens Rodentia e Didelphimorphia), representando 44\% do total de espécies desse grupo em território brasileiro (Paglia et al. 2012). Essas espécies estão distribuídas nas diversas formações que compõe o Cerrado, incluindo ambientes florestais com 
características distintas. Dentre essas fitofisionomias, estão as matas de galeria e os cerradões, os quais diferem em complexidade ambiental e previsibilidade de recursos. As matas de galeria possuem maior densidade de lianas e de dossel, bem como uma maior altura e ambientes mais estratificados verticalmente, configurando uma fitofisionomia mais complexa que o cerradão (Hannibal e Cáceres 2010). Além disso, os recursos em cerradão são potencialmente disponíveis mais sazonalmente para a fauna do que em mata de galeria (Melo et al. 2013), visto que essa última margeia cursos d'água geralmente perenes. Estas peculiaridades de cada fitofisionomia permitem a avaliação de possíveis diferenças na partição de nicho alimentar, na dieta e na estrutura trófica de conjuntos similares de espécies em cada formação florestal.

O modo como as espécies particionam o nicho em fitofisionomias distintas pode ser comparado por meio da avaliação dos requerimentos de hábitat, dieta e horários de atividade das espécies em cada formação (Schoener 1974). Alguns sistemas ecológicos possibilitam avaliar aspectos como requerimento de hábitat e dieta por meio da análise de isótopos estáveis (SIA, abreviatura do nome original Stable Isotopes Analysis). Isótopos estáveis de carbono $\left({ }^{13} \mathrm{C} /{ }^{12} \mathrm{C}\right)$ e nitrogênio $\left({ }^{15} \mathrm{~N} /{ }^{14} \mathrm{~N}\right)$ dos tecidos das espécies estudadas podem ser organizados de forma a gerar coordenadas em espaços multivariados, denominados $\delta$-espaços, que refletem dimensões análogas às do nicho hutchinsoniano (dimensões bionomic e scenopoetic) (Newsome et al. 2007). Os $\delta$ espaços podem ser usados como uma indicação do nicho realizado uma vez que a composição química dos tecidos dos indivíduos é fortemente influenciada pelo que o indivíduo consome e pelo hábitat que ele ocupa. Com base nisso, Newsome et al. (2007) propuseram o termo nicho isotópico como uma ferramenta para avaliar as características ecológicas dos organismos relevantes para o nicho ecológico.

A inferência de dieta por meio de isótopos estáveis funciona por meio da análise da assinatura isotópica de tecidos animais e posterior comparação com a assinatura de possíveis recursos utilizados pelas espécies em estudo. A assinatura isotópica do tecido animal é produto do que foi consumido e dos processos metabólicos envolvidos na assimilação desse substrato (BenDavid e Flaherty 2012). Além de possibilitar o cálculo de métricas relacionadas ao nicho isotópico, como sobreposição e largura de nicho (Layman et al. 2007; Jackson et al. 2011), a SIA permite 
avaliar a contribuição de possíveis recursos alimentares para a dieta das espécies (Phillips 2012), bem como verificar a diversidade e redundância trófica da comunidade (Layman et al. 2007, 2012).

A SIA é vantajosa com relação às metodologias clássicas de avaliação de dieta em pequenos mamíferos (ex. conteúdo estomacal, fezes) por indicarem o que foi de fato assimilado para formar o tecido analisado, além de refletir a dieta a longo prazo do indivíduo, a depender do tecido analisado (revisão em Crawford et al. 2008; Ben-David e Flaherty 2012). Entretanto, essa metodologia possui desvantagens quanto à falta de refinamento taxonômico, uma vez que dificilmente é possível identificar com acurácia os itens alimentares ingeridos, especialmente em ambientes complexos (Phillips e Gregg 2003; Ben-David e Flaherty 2012). Apesar disso, a SIA é uma ferramenta importante que complementa informações e compensa algumas desvantagens de outras metodologias de avaliação de dieta (Layman e Post 2008).

No presente estudo, investiguei a assimilação isotópica e partição de recursos alimentares por pequenos mamíferos não-voadores em dois tipos de florestas que ocorrem no Cerrado: cerradão e mata de galeria. Essa avaliação foi feita por meio de isótopos estáveis de ${ }^{13} \mathrm{C}$ e ${ }^{15} \mathrm{~N}$. Meus objetivos foram (i) caracterizar a assimilação isotópica das espécies nessas duas fitofisionomias e (ii) comparar a partição de nicho isotópico entre conjuntos similares de espécies presentes em cerradão e em mata de galeria. Considerando que a mata de galeria é uma fitofisionomia mais complexa que o cerradão e proporcionaria potencialmente uma maior amplitude de nichos ecológicos, minhas expectativas foram de uma maior amplitude total de nicho isotópico da comunidade de mata de galeria e maior especialização no uso do nicho pelas espécies nessa fitofisionomia. Em relação a variações entre ambientes no nicho isotópico de cada uma das espécies, como pequenos mamíferos em geral são considerados como generalistas em dieta, acredito que uma maior complexidade e maior estabilidade ao longo do ano nas matas de galeria levaria as espécies que ocorrem em ambos os tipos florestais a apresentarem uma maior amplitude de nicho isotópico no cerradão em comparação com estas matas. Isso porque esses animais poderiam compensar uma possível maior variação anual na disponibilidade de recursos em cerradão por meio de uma maior amplitude de recursos utilizados. 


\title{
Material e métodos
}

\author{
Sobre o Cerrado
}

O bioma Cerrado ocupa aproximadamente $23 \%$ do território brasileiro e consiste em um mosaico de fitofisionomias que variam desde formações abertas, savânicas até florestais (Ratter et al. 1997). Os principais determinantes do Cerrado são o clima, especialmente a precipitação, o solo e a dinâmica do fogo (Walker et al. 1987; Oliveira-Filho e Ratter 2002). As diversas combinações destes fatores geram heterogeneidade ambiental e consequentemente uma alta diversidade de espécies no bioma (Ratter et al. 1997). Apesar de sua importância, o Cerrado sofre degradação em uma taxa média de 1,1\% ao ano e estima-se que $48,2 \%$ a $55 \%$ do seu território já foi transformado para uso humano (Machado et al. 2004; MMA e IBAMA 2011). Esta acelerada perda de biodiversidade juntamente com o alto grau de endemismo e degradação caracteriza o bioma como um hotspot de biodiversidade (Mittermeier et al. 1998, 2011).

Em sua porção central, o Cerrado possui clima tropical chuvoso (Aw segundo a classificação de Köppen). Este é caracterizado por períodos bem definidos de seca entre abril e setembro, e de chuva entre outubro e março (Rizzini 1997), período que concentra cerca de 90\% da pluviosidade anual (Miranda et al. 1993). As temperaturas médias variam entre $22^{\circ}$ e $27^{\circ} \mathrm{C}$ e a precipitação anual média é de 1500 mm (Klink e Machado 2005).

Área de estudo

As áreas de estudo estavam localizadas no Jardim Botânico de Brasília (JBB) e na Fazenda Água Limpa (FAL) (Figura 1). Ambos estão inseridos na Área de Proteção Ambiental (APA) Gama e Cabeça de Veado no Distrito Federal que possui 23.650 ha de extensão (Unesco 2003). O Jardim Botânico de Brasília (JBB) possui 4.535 ha de extensão (IBRAM 2014) enquanto que a Fazenda Água Limpa, pertencente à Universidade de Brasília (UnB), possui aproximadamente 4.500 ha (FAL 2009). 
Realizei a coleta de dados nos meses de agosto, setembro e outubro de 2014 e janeiro, fevereiro e março de 2015 em quatro fragmentos naturais de hábitat: duas matas de galeria denominadas A $\left(15^{\circ} 57^{\prime} 14^{\prime \prime} S 47^{\circ} 57^{\prime} 44^{\prime \prime O}\right)$ e B $\left(15^{\circ} 57^{\prime} 38^{\prime \prime S} 47^{\circ} 56^{\prime} 37^{\prime \prime O}\right)$ e dois cerradões, C

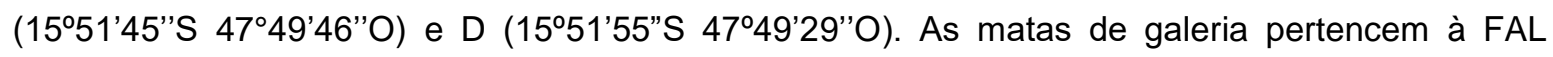
enquanto que os cerradões pertencem respectivamente ao JBB e à Escola de Administração Fazendária (ESAF), localizada ao lado do JBB.

O cerradão é uma formação florestal caracterizada por uma cobertura arbórea que pode oscilar entre $50 \%$ e $90 \%$, com dossel de 8 a $15 \mathrm{~m}$ de altura e presença de arbustos e herbáceas (Ribeiro e Walter 2008). A flora do cerradão consiste em espécies vegetais comuns ao cerrado sensu stricto e de espécies comuns à mata de galeria. Além disso, áreas de cerradão geralmente ocorrem em manchas associadas a solos de alta qualidade e são muito visadas pela agricultura (Unesco 2003). Já as matas de galeria formam faixas florestais ao longo de cursos d'água, possuindo dossel que atinge de 20 a 30m de altura e cobertura arbórea que varia entre $70 \%$ a 95\% (Ribeiro e Walter 2008). A flora associada a essas matas compreende espécies endêmicas, de cerrado sensu stricto, de cerradão e de outros biomas, como Mata Atlântica e Amazônica (Oliveira-Filho e Ratter 2002; Unesco 2003).

Coleta de dados

Amostragem de pequenos mamíferos

Realizei a amostragem dos pequenos mamíferos em conjunto com outros projetos que envolviam capturas desses animais, por esse motivo, o esforço amostral diferiu entre estações do ano. Coletei os dados durante duas campanhas de captura na estação chuvosa e duas na seca, com exceção das matas de galeria que, na estação chuvosa, foram amostradas em uma única campanha no mês de março. Nas matas de galeria, as armadilhas foram dispostas em duas transecções distantes $30 \mathrm{~m}$, cada uma com 30 estações de captura equidistantes $15 \mathrm{~m}$. No cerradão JB1, 
estabeleci três transecções equidistantes $15 \mathrm{~m}$, cada uma com 20 estações de captura, enquanto que no cerradão ESAF, dispus de quatro transecções, totalizando também 60 estações de captura. 


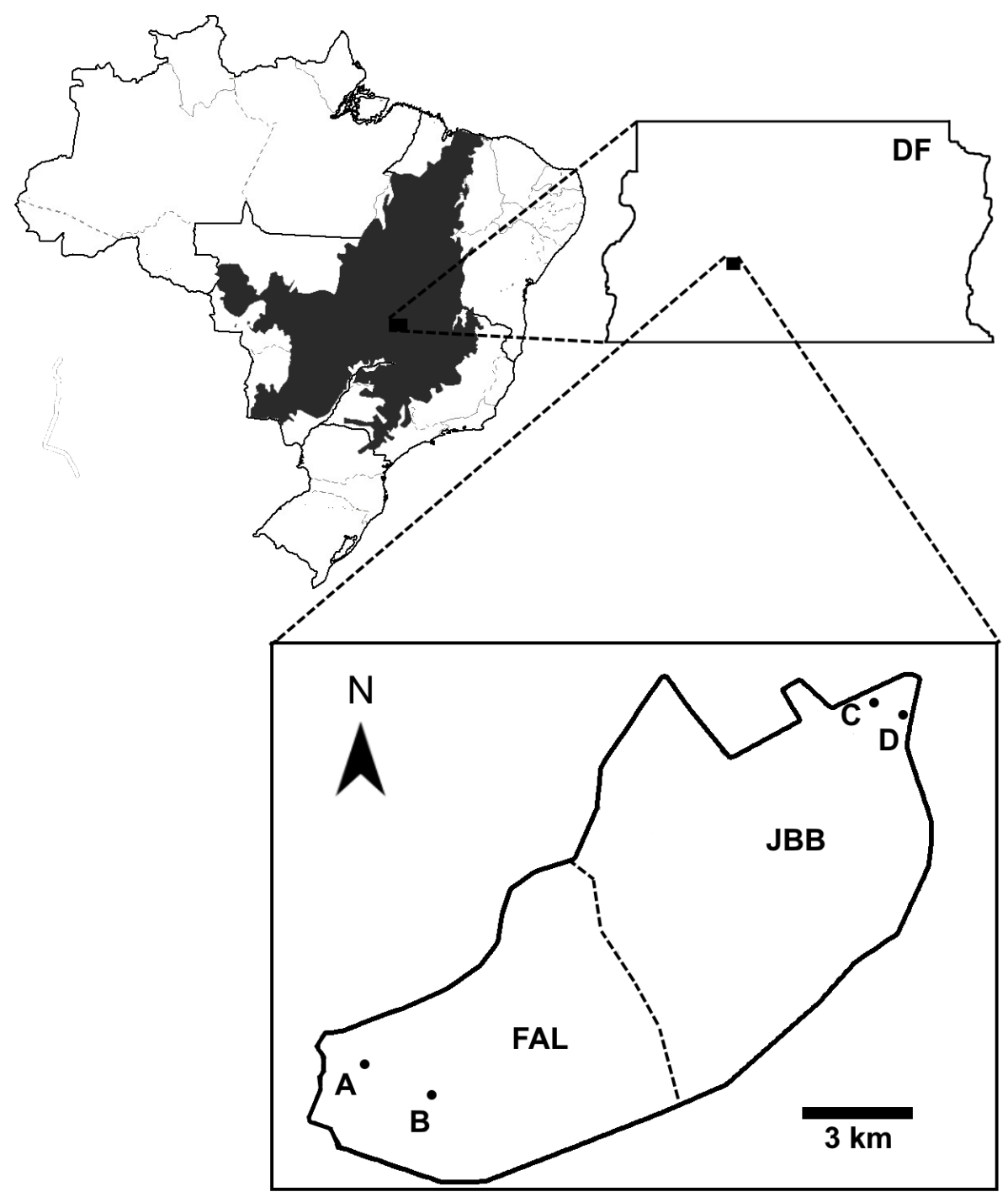

Figura 1. Localização das áreas de mata de galeria $(A$ e $B)$ e de cerradão ( $C$ e $D)$ amostradas na APA Gama e Cabeça-de Veado no Distrito Federal (DF), Brasil. As áreas de mata de galeria estão localizadas na Fazenda Água Limpa da Universidade de Brasília (FAL), o cerradão C está localizado no Jardim Botânico de Brasília (JBB) e o cerradão D na Escola de Administração Fazendária (ESAF). A área em cinza delimita a distribuição do bioma Cerrado. 
Em ambas fitofisionomias, amostrei os três estratos: solo, sub-bosque $(1-1,5 \mathrm{~m}$ acima do solo) e dossel ( 8m acima do solo). A extensão em dias e o número de armadilhas usadas em cada campanha diferiu entre as áreas, porém o esforço-amostral entre elas foi semelhante (Capetinga: 3286, Barragem: 3456, JB1: 3242, ESAF: 3584 armadilhas-noite). O esforço-amostral na estação seca foi de 9196 e na chuvosa de 4372, totalizando 13568 armadilhas-noite.

Capturei os indivíduos por meio de armadilhas Sherman $(11,0$ x 12,5 x 37,0 cm e 9,0 x 9,5 x 23,0 cm) e Tomahawk iscadas diariamente com uma mistura de fubá, banana, pasta de amendoim, óleo de fígado de bacalhau e essência de baunilha. Marquei os animais capturados com dois brincos (modelo 1005-1 da National Band and Tags Co), um em cada orelha. Também os identifiquei a nível de espécie, medi o comprimento do corpo (CC), da cauda (CA), da pata posterior direita (PP) da orelha $(\mathrm{OR})$ e pincei amostras de pelo para análise de isótopos estáveis, somente na primeira captura de cada indivíduo. Além disso, defini o sexo e a condição reprodutiva dos indivíduos bem como os pesei com o auxílio de um dinamômetro (Pesola ${ }^{\circledR}$, graduação 1g). Após os procedimentos, soltei os indivíduos na mesma estação da captura. As amostras de pelo refletem a assimilação isotópica desde a sua formação, uma vez que são tecidos metabolicamente inertes (Kelly 2000). Porém, o tempo necessário para que uma mudança de dieta seja verificada por isótopos estáveis nesse tecido é de 47,5 dias, pelo menos para gerbilos (Tieszen et al. 1983).

\section{Coleta de recursos alimentares}

Com o objetivo de estabelecer a assinatura isotópica de possíveis recursos utilizados por pequenos mamíferos (baseline) em cada área, coletei artrópodes, frutos, flores, briófitas e fungos por meio de uma campanha de coleta em setembro e outra em janeiro. Em cada campanha, aleatorizei 30 estações de captura nas quais instalei uma armadilha de queda (pitfall), uma armadilha de interceptação e queda (janela suspensa) e um coletor de frutos que permaneceram no local por 5 dias. 
Coleta de artrópodes

Realizei a coleta de artrópodes por meio das armadilhas de queda (pitfalls) e de interceptação e queda (janelas suspensas). Os pitfalls consistiram em copos descartáveis de 200ml enterrados no solo contendo cerca de $150 \mathrm{ml}$ de uma mistura a base de água e algumas gotas de detergente. As janelas foram feitas com garrafas PET de 2L com uma abertura de aproximadamente $17 \mathrm{~cm}$ de altura por $12 \mathrm{~cm}$ de largura contendo a mesma mistura das armadilhas de queda (metodologia modificada de Melo et al. 2001). As janelas eram suspensas por meio de uma corda a uma altura de aproximadamente $5 \mathrm{~m}$ para capturar principalmente artrópodes que utilizam os estratos superiores.

Peneirei o conteúdo de cada armadilha em campo (malha de $0,3 \mathrm{~mm}$ ) e o acondicionei em potes plásticos. No laboratório, lavei esse material sobre água corrente para retirada de sedimento, galhos e folhas. Posteriormente, triei o material para identificação dos artrópodes a nível de ordem e os classifiquei quanto à guilda alimentar quando possível (ex. fitófago, predador, generalista, hematófago, coprófago, nectarívoro).

Coleta de frutos e flores

Coletei frutos e flores por meio de coletores instalados ao lado das armadilhas para artrópodes. Este aparato consistiu em um tecido branco de $1 \mathrm{~m}^{2}$ esticado e suspenso por meio de quatro hastes de alumínio a uma altura de aproximadamente $1 \mathrm{~m}$ do chão, paralelo a este. No centro do tecido, fiz uma abertura circular para prender um pote plástico que serviu para armazenar os frutos e flores que caíam no tecido (metodologia em Camargo 2015).

Além de usar o coletor, recolhi frutos ao longo das transecções, bem como fungos e briófitas para compor o baseline das áreas. Identifiquei as amostras vegetais a nível de família quando foi possível. Dentre as amostras enviadas para análise, selecionei famílias de plantas mais consumidas por pequenos mamíferos segundo a literatura (Ellis et al. 1998; Cáceres 2004; Vanessa do Nascimento Ramos 2007; Lessa e da Costa 2008; Camargo et al. 2013), mais abundantes nas áreas 
e que estavam frutificando no período do estudo para estimativas de frugivoria dos pequenos mamíferos. Essas famílias foram as seguintes: Arecaceae, Cyperaceae, Euphorbiaceae, Fabaceae, Lauraceae, Melastomataceae, Myrtaceae, Piperaceae, Poaceae, Rubiaceae, Sapindaceae e Solanaceae.

Análise de isótopos estáveis

Preparei as amostras de pelos para análise de isótopos estáveis por meio de um protocolo de limpeza que consistia na lavagem com água destilada, seguida de imersão dos pelos em uma solução de duas partes de clorofórmio para um de metanol por 30 minutos e posterior lavagem com água destilada novamente (Cryan et al. 2012). Limpei as amostras de recursos alimentares usando somente água destilada. Posteriormente, sequei todas as amostras em estufa a $60^{\circ} \mathrm{C}$ por 3 (artrópodes e pelos) a 10 dias (demais amostras), as triturei e pesei em cápsulas de alumínio (mínimo de 1,5mg) usando balança analítica. As amostras foram enviadas ao Stable Isotope Facility da Universidade da California (Davis, EUA) para determinação das suas concentrações isotópicas de nitrogênio e carbono.

As composições isotópicas foram determinadas por meio de um espectrômetro de massa de razão isotópica de fluxo contínuo (PDZ Europa 20-20) acoplado ao analisador elemental (PDZ Europa ANCA-GSL). Os desvios padrão a longo prazo dos padrões internos utilizados no Stable Isotope Facility foram de 0,2\% para carbono e 0,3\% para nitrogênio (mais detalhes a respeito da metodologia do laboratório "http://stableisotopefacility.ucdavis.edu/13cand15n.html").

A razão de isótopos pesados e leves de carbono $\left({ }^{13} \mathrm{C} /{ }^{12} \mathrm{C}\right)$ e nitrogênio $\left({ }^{15} \mathrm{~N} /{ }^{14} \mathrm{~N}\right)$ foram calibrados respectivamente com as razões dos gases Vienna Peedee Belemnite $\left(\delta^{13} \mathrm{C}\right)$ e nitrogênio atmosférico $\left(\delta^{15} \mathrm{~N}\right)$, e expressos com relação ao padrão internacional por meio da notação:

$$
\delta X=\left(R_{\text {sample }} / R_{\text {standard }}-1\right) \times 1000
$$


em que $X$ se refere a ${ }^{13} \mathrm{C}$ ou ${ }^{15} \mathrm{~N}$ enquanto que $R_{\text {sample }}$ e $R_{\text {standard }}$ são as razões de isótopos pesados e leves de carbono ou nitrogênio da amostra e do padrão, respectivamente. Os resultados foram dados em notação isotópica (ס) e em partes por mil (\%).

Os valores de $\delta^{13} \mathrm{C}$ e $\delta^{15} \mathrm{~N}$ das amostras de pelo e de recursos informam a respeito da posição trófica das espécies na comunidade, bem como da diversidade e redundância trófica (Layman et al. 2007). Mais especificamente, as razões dos isótopos estáveis de carbono informam a principal fonte deste nutriente utilizada pela espécie, dentre plantas C3 ou C4. Essa discriminação é possível pelo enriquecimento de $12-14 \%$ nos valores de $\delta^{13} \mathrm{C}$ de plantas C4 com relação às C3 (Lajtha e Michener 1994). Por outro lado, os isótopos de nitrogênio esclarecem a posição trófica da espécie na comunidade (Post 2002). A avaliação desses aspectos é possível dado o enriquecimento de $\delta^{15} \mathrm{~N}$ (geralmente entre $2 \%$ e 4\%o) a cada nível trófico (Fry 2008).

\section{Análises estatísticas}

Com o objetivo de estimar a assimilação isotópica dos pequenos mamíferos, utilizei modelos de mistura de isótopos estáveis com base em análise Bayesiana. Essa abordagem estima a contribuição proporcional dos possíveis recursos para o tecido do consumidor (Parnell et al. 2010). Selecionei frutos e artrópodes como possíveis recursos alimentares e um fracionamento médio de 2,0\%o para $\delta^{13} \mathrm{C}$ (DeNiro e Epstein 1978) e de 3,2\% para $\delta^{15} \mathrm{~N}$ (DeNiro e Epstein 1981) a fim de estimar o nível de insetivoria (ou frugivoria) das espécies de pequenos mamíferos. Estimei a dieta de populações com no mínimo três amostras de pelos analisados, logo não obtive estas informações para as populações de Oligoryzomys nigripes $(n=2)$ e Cerradomys scotti $(n=1)$ em cerradão. Realizei essas análises por meio do pacote siar (Parnell et al. 2010) no software $R$ versão 3.2 .2 ( $R$ Development Core Team).

Além disso, avaliei aspectos da estrutura trófica das comunidades de cerradão e de mata de galeria por meio de seis métricas baseadas nos dados de $\delta^{13} \mathrm{C}$ e $\delta^{15} \mathrm{~N}$ z-transformados (Layman et al. 2007; Hoeinghaus e Zeug 2008): (i) amplitude de $\delta^{13} \mathrm{C}\left(\delta^{13} \mathrm{C}\right.$ range), que reflete a diversidade de 
recursos basais assimilados; (ii) amplitude de $\delta^{15} \mathrm{~N}\left(\delta^{15} \mathrm{~N}\right.$ range), uma medida de diversidade trófica da comunidade; (iii) área total (TA), que indica a amplitude de nicho; (iv) distância média para o centróide (CD), que descreve a amplitude de nicho e de espaçamento entre as espécies; (v) distância média do vizinho mais próximo (MNND), que reflete o empacotamento das espécies; e (vi) desvio padrão da distância do vizinho mais próximo (SDNND), que descreve a uniformidade deste empacotamento.

Essas métricas indicadas acima informam a respeito da diversidade trófica e posição relativa das espécies quando aplicadas a nível de comunidade, e descrevem a largura de nicho isotópico a nível de população. Entretanto, falham em considerar variações naturais do sistema. Portanto, optei por calcular as métricas a nível de comunidade utilizando técnicas de inferência Bayesianas que propagam incertezas e permitem comparações mais robustas entre comunidades diferentes (para mais detalhes, ver Jackson et al. 2011). Para o cálculo destas métricas, selecionei espécies para as quais houve no mínimo pelos de três indivíduos analisados. Logo, não incluí O. nigripes em ambas fitofisionomias e C. scotti. Para comparação, calculei também, para cada fitofisionomia, as métricas de nicho isotópico baseadas nos dados de $\delta^{13} \mathrm{C}$ e $\delta^{15} \mathrm{~N}$ já citadas anteriormente, só que considerando as médias dos valores de cada uma das espécies capturadas, conforme sugerido por Layman et al. (2007), independentemente do número de amostras para cada espécie. Esses cálculos foram feitos considerando todas as espécies capturadas em cada uma das comunidades e também considerando somente os pequenos mamíferos capturados tanto em cerradão quanto em mata de galeria.

Ademais, por conta da sensibilidade dessa abordagem ao tamanho amostral, optei por calcular a largura de nicho de cada espécie por meio de métricas baseadas em elipses multivariadas estimadas por inferência Bayesiana corrigidas para pequenos tamanhos amostrais (SEAc: corrected standard ellipse area) em detrimento da métrica TA (área total) (Jackson et al. 2011). Para o cálculo destas métricas (SEAc), selecionei espécies para as quais houve no mínimo pelos de três indivíduos analisados. No caso das espécies $R$. macrurus e $D$. albiventris, cujo número de indivíduos diferiu muito entre as fitofisionomias, optei por aleatorizar subconjuntos de tamanho igual ao da comunidade com menos indivíduos e calcular os valores de SEAc para cada subconjunto aleatorizado (1000 aleatorizações) com a indicação da variação desses valores obtidos com as aleatorizações. Calculei 
estas métricas por meio do pacote SIBER (Jackson et al. 2011) no software $R$ versão 3.2.2 ( $R$ Development Core Team).

\section{Resultados}

Obtive 852 capturas de 413 indivíduos pertencentes a nove espécies, três marsupiais e seis roedores, resultando em um sucesso de captura de 3,04\% (Tabela suplementar 1). Realizei a análise isotópica de 167 indivíduos de pequenos mamíferos, 93 indivíduos de artrópodes, 90 amostras de material vegetal (flores, frutos e briófitas) e 13 amostras de fungos. Em cerradão, as médias dos valores isotópicos obtidos para a comunidade de pequenos mamíferos foram de $-22.96 \%$ para $\delta^{13} \mathrm{C}$ (amplitude: $-24,52 \%$, $-9,99 \%$ ) e 5,29\% para $\delta^{15} \mathrm{~N}$ (amplitude: 1,75\%, 9,95\%०). Já na mata de galeria, essas médias foram de $-23,58 \%$ para $\delta^{13} \mathrm{C}$ (amplitude: $-26,52 \%$, $-11,07 \%$ ) e $5,71 \%$ para $\delta^{15} \mathrm{~N}$ (amplitude: 2,28\%o, 8,12\%o).

As espécies de ambas fitofisionomias se diferenciaram quanto à proporção em que ingerem frutos ou artrópodes (Figura 2). No geral, as espécies se dividiram em três categorias quanto à assimilação isotópica: (i) maior proporção de artrópodes assimilados; (ii) maior proporção de frutos ou sementes (C3 ou C4); e (iii) posição intermediária, que assimilam ambos os grupos em proporções similares. Os marsupiais Didelphis albiventris e Gracilinanus agilis assimilaram uma proporção maior de artrópodes que as demais espécies podendo ser classificados como insetívoros-

onívoros. Já o grupo de frugívoros-onívoros foi representado pelos roedores Oecomys bicolor e Oligoryzomys nigripes enquanto que as demais espécies (Caluromys lanatus, Hylaeamys megacephalus, Rhipidomys macrurus e Proechimys roberti) apresentaram uma posição intermediária. As espécies $O$. nigripes e $O$. bicolor foram as únicas cujos valores isotópicos para $\delta^{13} \mathrm{C}$ indicaram uma dieta baseada tanto em recursos C3 quanto C4 (Figuras 3 e 4). Não foi possível estimar a assimilação isotópica de artrópodes e frutos para $C$. scotti, por somente um indivíduo ter sido capturado. Porém, a assinatura isotópica deste indivíduo indica que ele consumiu recursos de origem C3 e C4 ou somente C4 (Figuras 3 e 4). 
A assimilação isotópica dos artrópodes diferiu entre as fitofisionomias. Em cerradão, houve maior diferenciação entre artrópodes fitófagos e predadores no eixo do $\delta^{15} \mathrm{~N}$, enquanto que em mata de galeria, esses grupos apresentaram maior sobreposição de nicho isotópico (Figuras 3 e 4). Os artrópodes generalistas, representados pelas ordens Blataria, Diptera e Orthoptera, apresentaram alta amplitude de $\delta^{15} \mathrm{~N}$ em ambas fitofisionomias. A assinatura isotópica de $\delta^{13} \mathrm{C}$ de todos os indivíduos indicou o consumo de recursos de origem de plantas C3 e de mesmo sinal isotópico das plantas analisadas tanto em cerradão quanto em mata de galeria (Figuras 3 e 4).

A assimilação isotópica de uma mesma espécie de mamífero em cada fitofisionomia foi semelhante (Figuras 3 e 4). Entretanto, a diferenciação alimentar entre as espécies de cerradão foi maior. Em mata de galeria, os marsupiais não apresentaram uma proporção de artrópodes tão alta quanto em cerradão e as espécies intermediárias assimilaram materiais de origem vegetal e animal em proporções mais similares. Além disso, o tamanho do nicho isotópico ocupado pelas espécies diferiu entre as fitofisionomias. Em mata de galeria, os marsupiais $G$. agilis e $D$. albiventris apresentaram valores de SEAc menores que em cerradão, enquanto que roedores como $H$. megacephalus, R. macrurus e O. bicolor expressaram valores maiores de SEAc nessa fitofisionomia (Figura 5).

A comunidade de pequenos mamíferos de cerradão apresentou uma maior amplitude de $\delta^{15} \mathrm{~N}\left(\delta^{15} \mathrm{~N}\right.$ range), bem como maiores valores de TA, CD, NND e SDNND que a mata de galeria (Figura 6). Ou seja, as espécies em cerradão apresentaram maior área de nicho isotópico, menor sobreposição de nicho isotópico e se distanciaram mais do centroide da comunidade, enquanto que as espécies em mata de galeria estiveram mais empacotadas (maior sobreposição). Não houve diferença aparente entre as fitofisionomias com relação à amplitude de $\delta^{13} \mathrm{C}\left(\delta^{13} \mathrm{C}\right.$ range). Valores maiores de TA para a comunidade de Cerradão foram encontrados também quando considerei todas as espécies das comunidades e quando considerei somente as espécies capturadas em ambas as fitofisionomias (Tabela suplementar 2). 

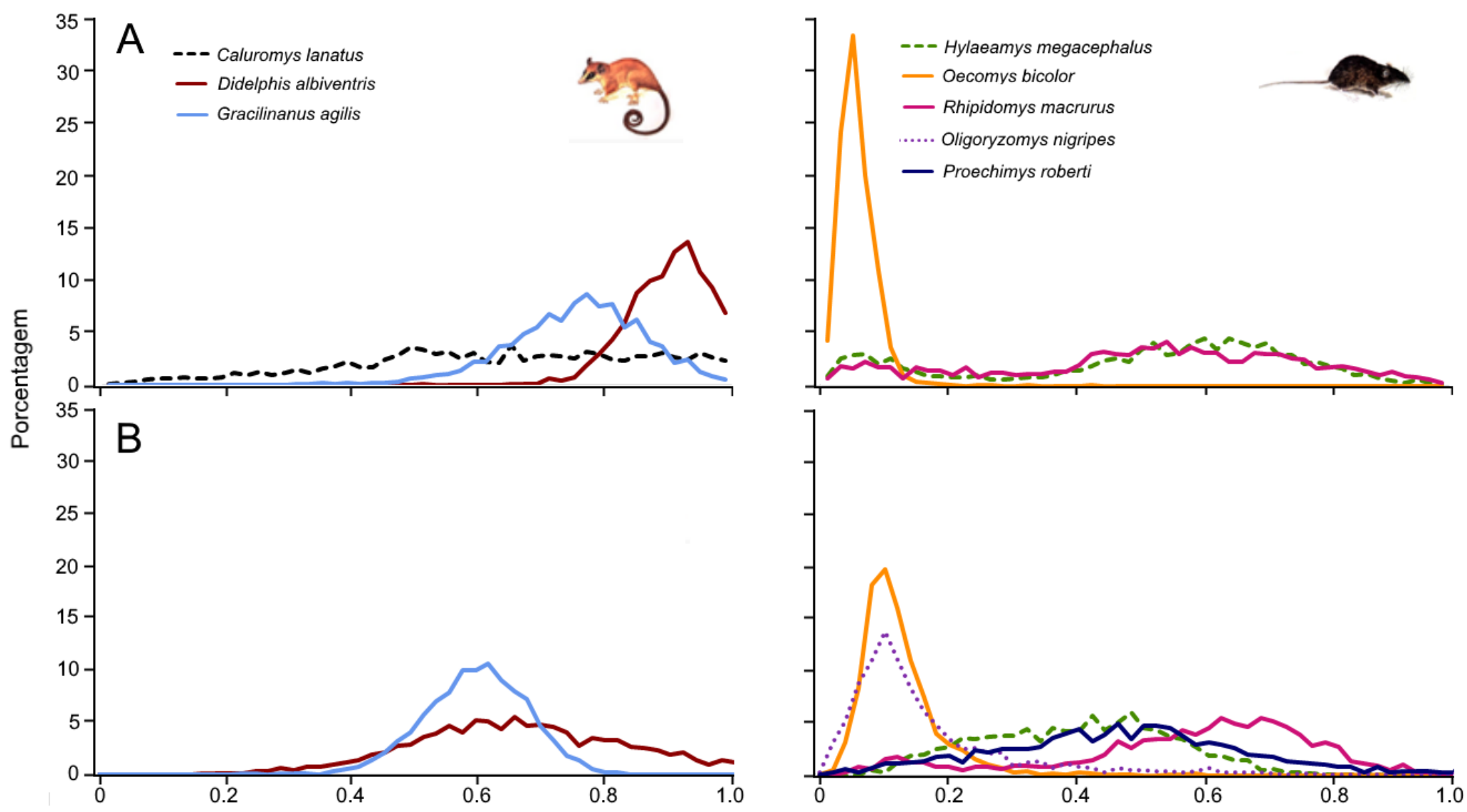

Proporção estimada de artrópodes

Figura 2. Estimativa da proporção de artrópodes consumidos pelas espécies de marsupiais Caluromys lanatus, Didelphis albiventris, Gracilinanus agilis e dos roedores Hylaeamys megacephalus, Oecomys bicolor, Rhipidomys macrurus, Oligoryzomys nigripes e Proechimys roberti em áreas de cerradão $(A)$ e de mata de galeria $(B)$ no Cerrado brasileiro, por meio da assinatura isotópica de $\delta^{13} \mathrm{C}$ e $\delta^{15} \mathrm{~N}$ de amostras de pelos. 


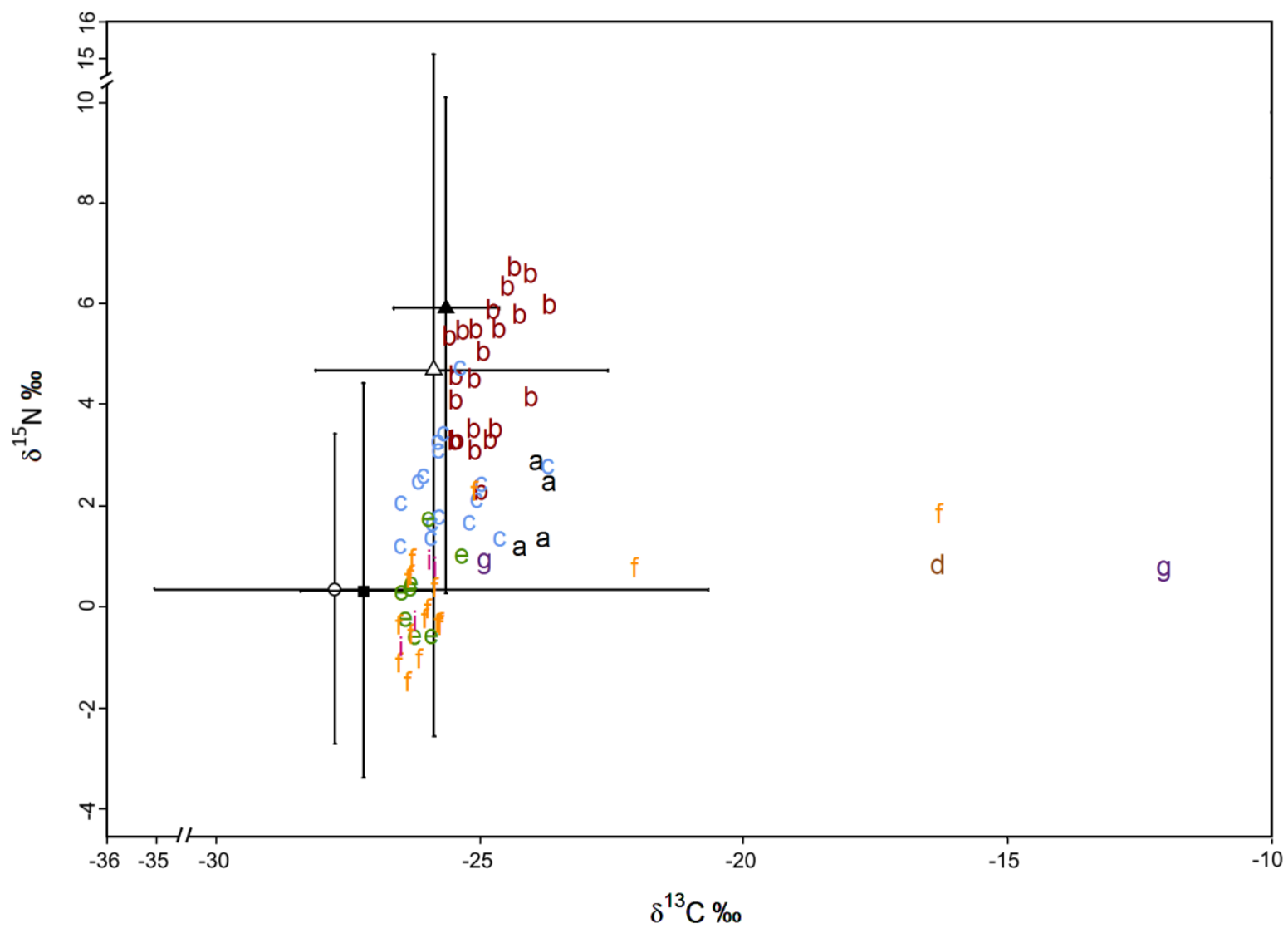

Figura 3. Assinatura isotópica de $\delta^{13} \mathrm{C}$ e $\delta^{15} \mathrm{~N}$ das espécies de pequenos mamíferos capturadas em áreas de cerradão no Cerrado do Brasil central. Códigos: marsupiais Caluromys lanatus (a), Didelphis albiventris (b), Gracilinanus agilis (c); roedores Cerradomys scotti (d), Hylaeamys megacephalus (e), Oecomys bicolor (f), Oligoryzomys nigripes (g) e Rhipidomys macrurus (i); possíveis recursos consumidos pelos mamíferos: frutos (círculo aberto), artrópodes fitófagos (quadrado fechado), artrópodes predadores (triângulo fechado) e artrópodes generalistas (triângulo aberto). 


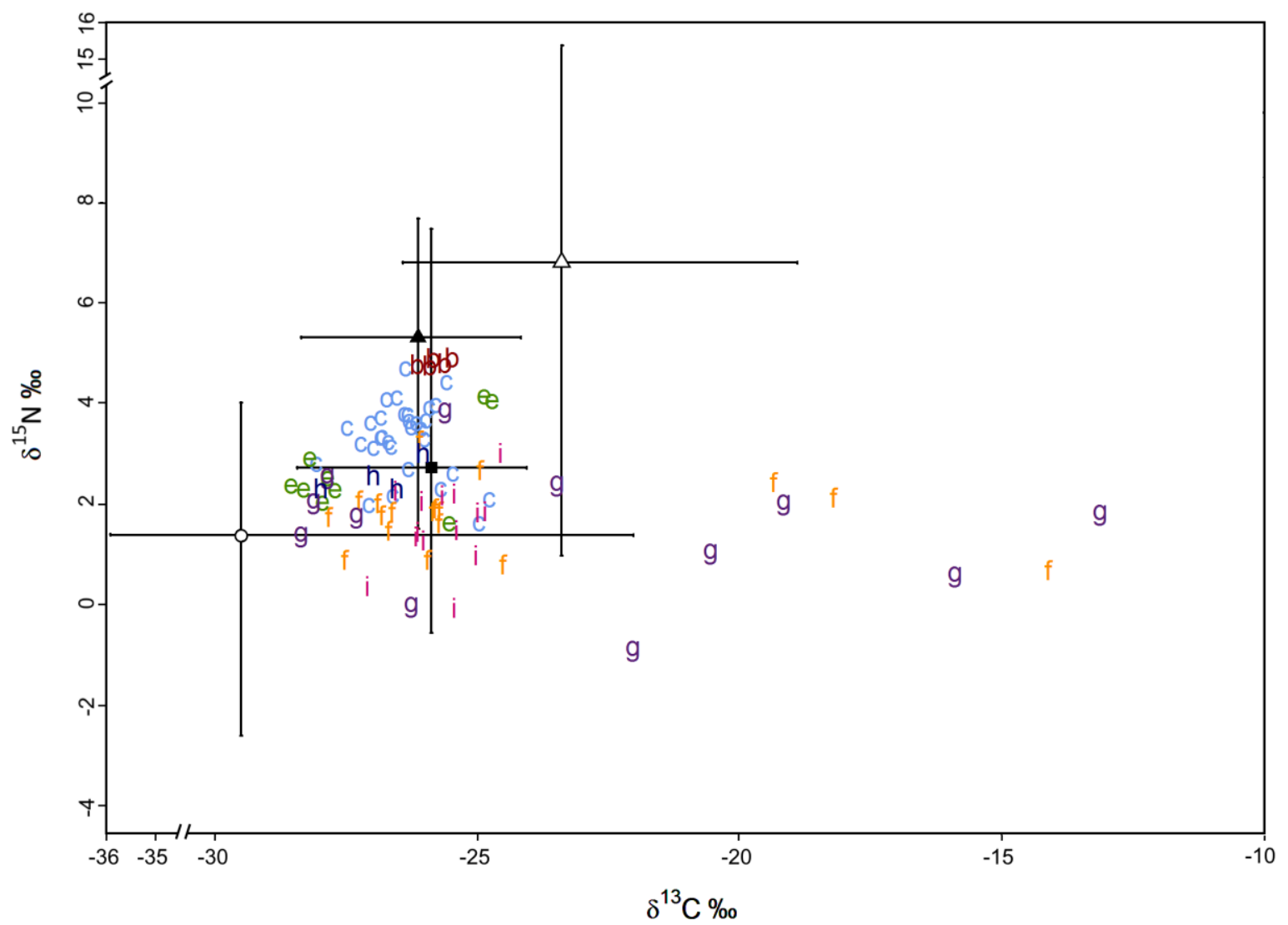

Figura 4. Assinatura isotópica de $\delta^{13} \mathrm{C}$ e $\delta^{15} \mathrm{~N}$ das espécies de pequenos mamíferos capturadas em áreas de mata de galeria no Cerrado do Brasil central. Códigos: marsupiais Didelphis albiventris (b), Gracilinanus agilis (c); roedores Hylaeamys megacephalus (e), Oecomys bicolor (f), Oligoryzomys nigripes (g), Proechimys roberti (h) e Rhipidomys macrurus (i); possíveis recursos consumidos pelos mamíferos: frutos (círculo aberto), artrópodes fitófagos (quadrado fechado), artrópodes predadores (triângulo fechado) e artrópodes generalistas (triângulo aberto). 
A)
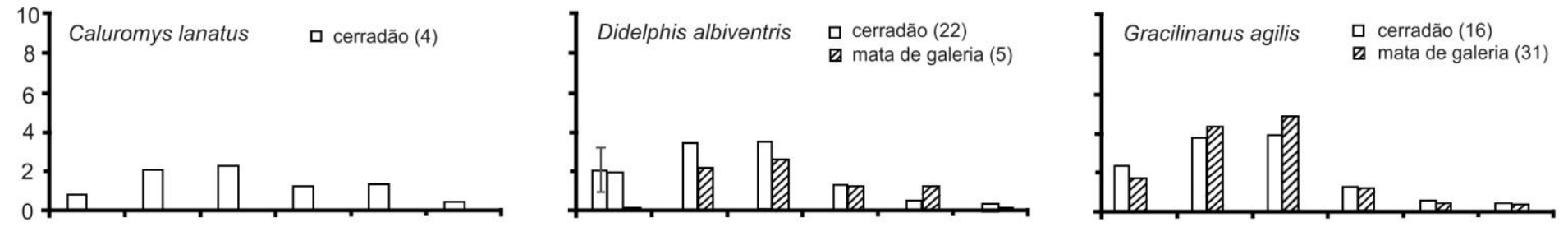

B)
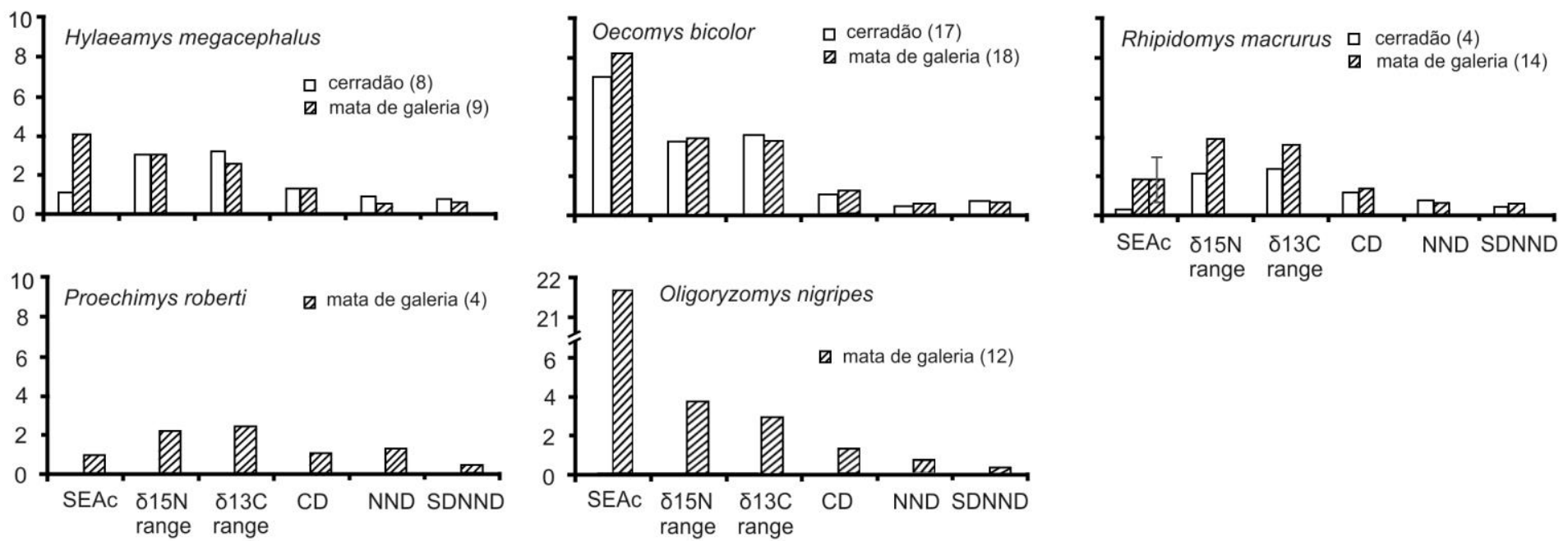

Figura 5. Métricas de nicho isotópico SEAc (corrected standard ellipse area), amplitude de $\delta^{15} \mathrm{~N}$, amplitude de $\delta^{13} \mathrm{C}$, distância média para 0 centroide (CD), distância média para o vizinho mais próximo (NND), desvio padrão da distância para o vizinho mais próximo (SDNND) (para maiores informações sobre as métricas, ver Layman et al. 2007; Jackson et al. 2011) para as espécies dos marsupiais (A) dos roedores (B) em dois tipos florestais no Brasil central, cerradão (barras brancas) e mata de galeria (barras preenchidas). 


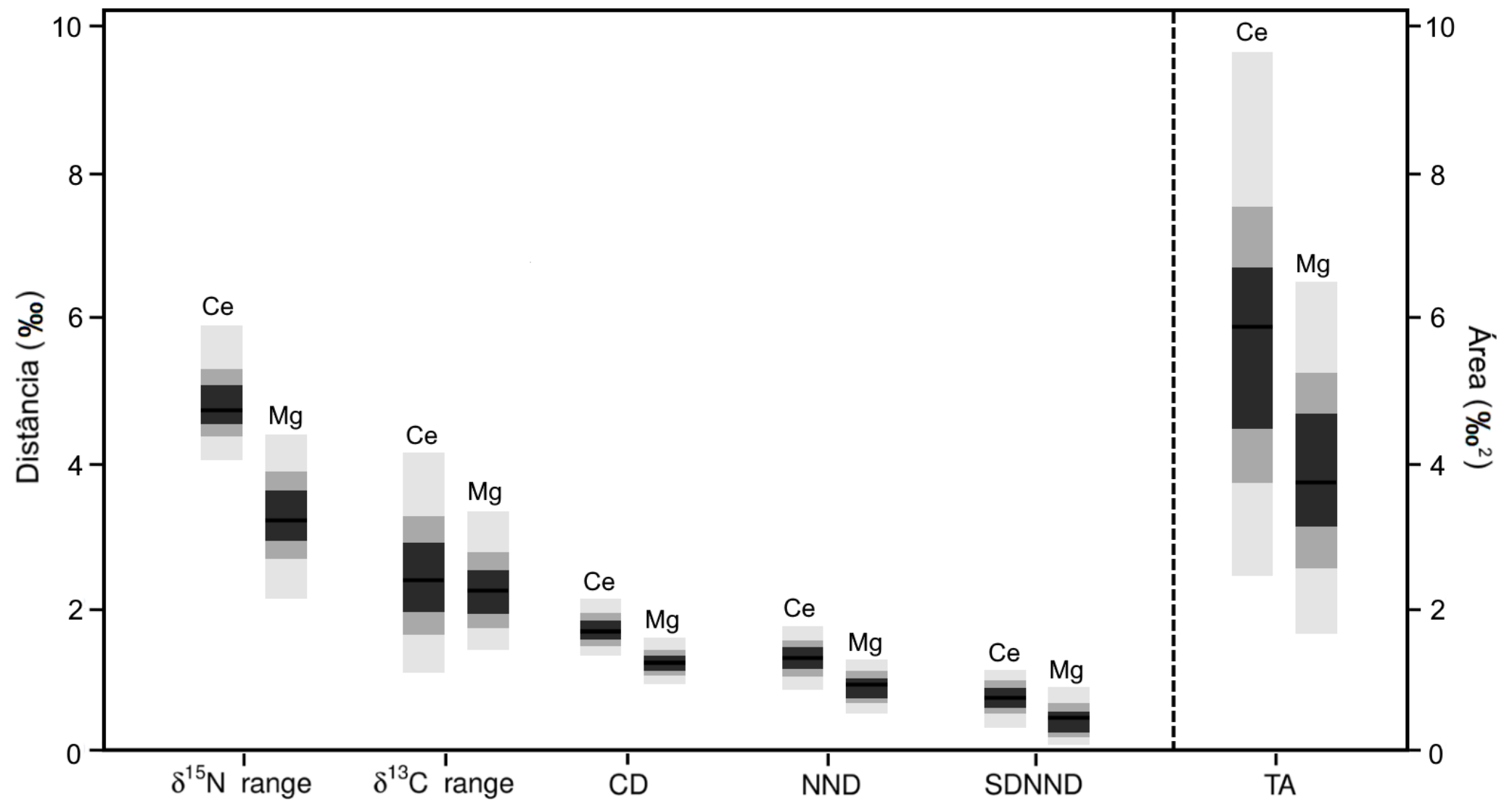

Figura 6. Métricas de nicho isotópico (Layman et al. 2007; Jackson et al. 2011) para comunidades de pequenos mamíferos em cerradão (Ce) e mata de galeria $(\mathrm{Mg})$. 


\section{Discussão}

No presente estudo, apresento uma caracterização até então inédita do nicho isotópico das comunidades de pequenos mamíferos em duas fitofisionomias florestais do Cerrado. As espécies em ambas fitofisionomias se diferenciaram quanto à proporção em que assimilam alimentos de origem vegetal (frutos, sementes) e de origem animal (artrópodes, pequenos vertebrados). A predominância de dietas menos especializadas entre pequenos mamíferos neotropicais também foi constatada em estudos que avaliaram a dieta por meio do conteúdo estomacal ou fezes (Meserve et al. 1988; Ellis et al. 1998; Campos et al. 2001; Cáceres 2002; Casella e Cáceres 2006; Talamoni et al. 2008).

Embora ambos os grupos tenham demostrado assimilar tanto recursos de origem animal quanto vegetal, detectei diferenças na assimilação isotópica entre as ordens de mamíferos estudadas. Os marsupiais ( $G$. agilis, $D$. albiventris, $C$. lanatus) apresentaram um $\delta^{15} \mathrm{~N}$ mais elevado que os roedores (demais espécies), sugerindo uma dieta mais insetívora. Esse padrão não era esperado uma vez que frutos constituem importantes fontes alimentares para essas espécies, principalmente para o marsupial C. lanatus, considerado frugívoro-onívoro na literatura (Cáceres 2002; Casella e Cáceres 2006; Camargo et al. 2011; Paglia et al. 2012). A provável ingestão de vertebrados por D. albiventris e C. lanatus (Camargo et al. no prelo; Cáceres 2002; Casella e Cáceres 2006), mesmo em menores proporções, e o consumo de invertebrados por G. agilis contribuem para a maior diferenciação entre marsupiais e roedores. A maioria dos marsupiais sul-americanos são insetívoro-onívoros (Cáceres 2004; Martins e Bonato 2004; Nowak 2005; Lessa e da Costa 2008; Bocchiglieri et al. 2010; Paglia et al. 2012), ao passo que os roedores neotropicais geralmente dependem mais de recursos como frutos e sementes que os marsupiais insetívoros (Fonseca e Kierulff 1988; Talamoni et al. 2008; Paglia et al. 2012). Essas diferenças na utilização de recursos alimentares podem ser explicadas pelas diferenças morfológicas e fisiológicas entre esses grupos (Nowak 2005; Samuels 2009; Santori et al. 2012).

As espécies que ocorreram simultaneamente em cerradão e mata de galeria apresentaram assimilação isotópica semelhantes em ambas fitofisionomias. No entanto, o padrão de variação 
observado quanto à amplitude de nicho isotópico (SEAc) foi inverso na comparação entre roedores e marsupiais, o que corroborou parcialmente a minha expectativa. Roedores apresentaram maior amplitude de nicho isotópico em matas de galeria enquanto que marsupiais apresentaram menor amplitude de nicho nessas matas comparado com o cerradão (Figura 4). Os padrões distintos entre esses dois grupos podem indicar uma diferença no grau de especialização, com os roedores sendo mais generalistas e com maior plasticidade na dieta (Landry 1970). Dessa forma, a maior amplitude de nicho dos roedores em matas pode ser explicado pela ingestão de uma maior diversidade de recursos nessa fitofisionomia (Baugh et al. 2004; Bearhop et al. 2004; Semmens et al. 2009) Além disso, a maior sobreposição de nicho isotópico da comunidade e amplitude desses roedores em mata de galeria pode estar relacionada com a maior disponibilidade de recursos nessa fitofisionomia e de como esse grupo generalista responde a essa disponibilidade (Meserve et al. 1988; Melo et al. 2013; Camargo 2015; Ribeiro 2015). Já os marsupiais poderiam se aproveitar de uma maior disponibilidade e variabilidade de recursos na mata para se alimentar mais seletivamente de invertebrados, que provavelmente compõe a dieta ótima para esse grupo por serem altamente proteicos (Vieira e Moraes 2003). Uma assimilação isotópica mais especializada em épocas de maior disponibilidade de recursos já foi reportada para pequenos mamíferos africanos (Codron et al. 2015). Além disso, Ribeiro (2015) também observou que o roedor $R$. macrurus apresentou maior seletividade alimentar sob condição de maior disponibilidade e variedade de recursos.

Em cerradão, a separação entre roedores e marsupiais no espaço de nicho isotópico foi maior e isso possibilitou a classificação de guildas alimentares mais evidentes nessa fitofisionomia. Os indivíduos de $D$. albiventris apresentaram os maiores valores de $\delta^{15} \mathrm{~N}$, indicando uma dieta com maior proporção de alimentos de origem animal. A proporção de artrópodes estimada na dieta dos indivíduos em cerradão foi de aproximadamente $90 \%$, enquanto que em mata de galeria esse valor foi cerca de $65 \%$. A dieta desse marsupial, inferida com base em análises fecais é caracterizada como onívora ou insetívora-onívora e constitui principalmente de vertebrados (30\%), lixo doméstico (30\%), invertebrados (20\%) e material vegetal (20\%) (Cáceres 2002; Vieira e Moraes 2003; Wilman et al. 2014). Entretanto, alguns autores classificam essa espécie como frugívora-onívora e oportunista no consumo de invertebrados e vertebrados (Paglia et al. 2012; Santori et al. 2012). Os 
valores elevados de $\delta^{15} \mathrm{~N}$ e de proporção de material animal na dieta podem ser devidos ao consumo de vertebrados e de lixo doméstico, recursos não avaliados neste trabalho. Apesar disso, essas estimativas estão de acordo com as encontradas em outros trabalhos.

O marsupial G. agilis apresentou assimilação isotópica com a segunda maior proporção de material de origem animal (60\% em mata de galeria e $75 \%$ em cerradão), seguido do marsupial $C$. lanatus. A assimilação isotópica de G. agilis indica uma dieta insetívora-onívora, o que está de acordo com o encontrado em outros trabalhos (Lessa e da Costa 2008; Bocchiglieri et al. 2010; Camargo et al. 2013). Sua dieta consiste principalmente de artrópodes das ordens Isoptera, Coleoptera e Hemiptera e frutos de Melastomataceae (Bocchiglieri et al. 2010; Camargo et al. 2011, 2013; Wilman et al. 2014). Por outro lado, a assinatura isotópica de C. lanatus indicou um consumo maior de frutos que os outros marsupiais (Figura 2). Apesar disso, a estimativa de insetivoria de $C$. lanatus encontrada nesse estudo foi mais alta do que o esperado. De acordo com as informações disponíveis na literatura, esse marsupial é frugívoro-onívoro, ocasionalmente consumindo invertebrados e vertebrados (Camargo et al., no prelo; Casella e Cáceres 2006; Paglia et al. 2012). Aproximadamente $70 \%$ da sua dieta consiste de frutos, sementes e plantas, $20 \%$ de invertebrados e 10\% de vertebrados (Wilman et al. 2014). Meus resultados quanto à dieta de $C$. lanatus não foram tão conclusivos quanto para os outros marsupiais por conta da alta variação na estimativa de grau de insetivoria (Figura 2). Esses resultados não indicaram, porém, uma dieta frugívora-onívora (Figuras 2 e 3). O consumo ocasional de vertebrados e invertebrados possivelmente influenciou o valor de $\delta^{15} \mathrm{~N}$ desse marsupial, tornando-o alto. Dessa forma, o consumo de vertebrados e invertebrados mesmo esporadicamente configura uma importante fonte de proteína assimilada por esse marsupial.

Em relação aos roedores, $R$. macrurus, $H$. megacephalus e $P$. roberti apresentaram estimativas intermediárias de proporção de artrópodes na dieta, dependendo mais de alimentos de origem vegetal que os marsupiais (Fig. 2). Informações sobre as dietas de roedores no geral são escassas, porém R. macrurus, $H$. megacephalus e $P$. roberti são classificados na literatura como frugívoros/predadores de sementes (Ferreira et al. 2011; Paglia et al. 2012). As posições tróficas de R. macrurus e H. megacephalus foram similares aos encontrados por Ribeiro (2015), que também 
avaliou a assimilação isotópica de pequenos mamíferos em outra região do Cerrado considerou que essas espécies seriam mais frugívoras. No presente estudo, houve uma considerável sobreposição dos valores isotópicos de frutos e artrópodes, o que dificultou a estimativa de insetivoria dessas espécies. Entretanto, é evidente que esses roedores dependem mais de alimentos de origem vegetal (frutos e sementes) que os marsupiais, principalmente $G$. agilis e $D$. albiventris.

As espécies $O$. nigripes e $O$. bicolor foram as únicas cujas assinaturas isotópicas indicaram o consumo de alimentos provenientes de fontes C3 e C4 (Fig. 3), ou seja, além de consumirem frutos de origem C3, consomem sementes de gramíneas C4 ou artrópodes que se alimentam dessas gramíneas. Em mata de galeria, gramíneas $\mathrm{C} 4$ se encontram principalmente em clareiras dentro dos fragmentos ou em bordas, onde a incidência de luz solar é mais intensa. Portanto, esses roedores em mata de galeria provavelmente se locomovem e consomem recursos provenientes dessas áreas. Outra possível explicação para o sinal C4 nos pelos desses indivíduos seria a locomoção para as áreas de campo adjacentes às matas de galeria. O. nigripes é uma espécie generalista em uso de habitat, podendo ocorrer em campos, savanas e florestas (Mares et al. 1986; Stallings 1989; Vieira e Marinho-Filho 1998) e que consome principalmente alimentos de origem vegetal, como sementes e folhas (Talamoni et al. 2008). Vieira e Marinho-Filho (1998) avaliaram o uso do habitat em mata de galeria e campo adjacente à mata por pequenos mamíferos e verificaram que $O$. nigripes transita entre as duas fitofisionomias. Além disso, essa espécie esteve associada a maior densidade de vegetação a $1 \mathrm{~m}$ de altura e menor densidade de árvores em área de Floresta com Araucária, indicando uma preferência por clareiras na mata (Dalmagro e Vieira 2005). Dessa forma, o sinal C4 evidente nos pelos dos indivíduos dessa espécie pode ser proveniente de material vegetal (folhas, sementes) de gramíneas nas áreas de campo adjacentes ou de clareiras (Galetti et al. 2016).

As espécies $O$. nigripes e $O$. bicolor apresentaram uma assimilação isotópica maior de alimentos de origem vegetal. Outros trabalhos verificaram que essas espécies são frugívoras/predadoras de sementes, sendo a dieta de $O$. bicolor composta totalmente por recursos vegetais, enquanto que a de 0 . nigripes consiste em $70 \%$ de recursos vegetais e $30 \%$ de invertebrados, semelhante às minhas estimativas (Talamoni et al. 2008; Paglia et al. 2012; Wilman et al. 2014; Ribeiro 2015). Entretanto, nas matas de galeria avaliadas por Ribeiro (2015), os 
indivíduos de $\mathrm{O}$. bicolor não consumiram gramíneas ou artrópodes com sinal C4, mesmo estas fontes sendo abundantes nas áreas de estudo. As matas de galeria avaliadas no presente estudo e no de Ribeiro (2015) diferem quanto ao grau de perturbação, sendo as últimas mais estreitas e perturbadas devido a incêndios recorrentes. Logo, é possível que o grau de conservação da mata de galeria possa influenciar o uso do espaço e a dieta de O. bicolor.

Meus resultados indicaram que os pequenos mamíferos em mata de galeria apresentaram uma amplitude de nicho isotópico menor em comparação com o cerradão, contrariando as minhas expectativas. A maior amplitude de nicho em cerradão se manteve independentemente do método utilizado e também quando a análise se restringiu às espécies comuns a ambas formações. Essa diferença foi devida principalmente à maior amplitude do $\delta^{15} \mathrm{~N}$ no cerradão, além da menor sobreposição de nicho entre as espécies dessa fitofisionomia (Figura 5). A conformação no espaço de nicho isotópico dos artrópodes também seguiu este mesmo padrão quanto a amplitude de $\delta^{15} \mathrm{Ne}$ sobreposição de nicho (Figura 3). Isso pode indicar que as diferenças na assimilação isotópica de pequenos mamíferos e de artrópodes entre as fitofisionomias são devidas às diferenças nas assinaturas isotópicas das plantas em cada tipo florestal. Apesar de ser menos complexo estruturalmente, o cerradão é mais heterogêneo e pode apresentar diversidade maior de plantas isotopicamente distintas que mata de galeria. Essa diversidade, por sua vez, reflete na assinatura isotópica de invertebrados e de pequenos mamíferos que se alimentam tanto de plantas quanto de invertebrados. Symes et al. (2013) avaliaram o nicho isotópico de pequenos mamíferos em um mosaico de campos e florestas na África e verificaram menor amplitude de nicho isotópico em florestas devido à diversidade de plantas isotopicamente mais distintas nas áreas de campo.

$\mathrm{O} \delta^{15} \mathrm{~N}$ foliar de plantas em ambientes limitados por nitrogênio como o cerrado s.S., por exemplo, difere de plantas em florestas tropicais com alta disponibilidade de nitrogênio (Martinelli et al. 1999; Bustamante et al. 2004). Em savanas, a amplitude de $\delta^{15} \mathrm{~N}$ nas folhas e no solo é maior e muitas espécies de plantas, especialmente leguminosas, apresentam valores extremamente baixos de $\delta^{15} \mathrm{~N}$, o que contribui para essa diversidade de valores (Schmidt e Stewart 2003; Bustamante et al. 2004). Por outro lado, florestas tropicais com alta disponibilidade de nitrogênio possuem plantas mais enriquecidas em $\delta^{15} \mathrm{~N}$ foliar e com valores mais constantes (Högberg e Alexander 1995; 
Martinelli et al. 1999; Schmidt e Stewart 2003; Bustamante et al. 2004). No presente trabalho, os valores de $\delta^{15} \mathrm{~N}$ de frutos coletados em cerradão variaram de $-4,70$ a 5,61\%o (média: 0,10\%o), enquanto que os valores em mata de galeria variaram entre $-2,60$ a 4,01\%o (média: 1,07\%o). Ou seja, apesar de um valor médio bem mais alto para a mata de galeria, o cerradão apresentou uma maior amplitude de variação nesses valores. Embora não tenha coletado amostras de solo e folhas de diversas espécies de plantas, é possível que o cerradão apresente maior amplitude de $\delta^{15} \mathrm{~N}$ que a mata de galeria, similar às diferenças existentes entre ambientes savânicos limitados por nitrogênio e florestas com alta disponibilidade desse componente. Por sua vez, a maior amplitude de $\delta^{15} \mathrm{~N}$ das plantas em cerradão pode explicar o maior espaçamento no eixo do $\delta^{15} \mathrm{~N}$ das espécies de pequenos mamíferos e artrópodes de cerradão.

As estimativas de amplitude e sobreposição de nicho utilizando a SIA se mostraram adequadas em diversos sistemas (Newsome et al. 2009; Rodríguez e Gerardo Herrera 2013; Symes et al. 2013; Codron et al. 2015; Ribeiro 2015) e tem sido usadas por muitos autores na investigação da partição de recursos, apesar das ressalvas a respeito da metodologia (Layman et al. 2007; Newsome et al. 2007, 2012; Hoeinghaus and Zeug 2008; Layman and Post 2008; Jackson et al. 2011). No presente estudo, foi possível compreender a assimilação isotópica das espécies, e identificar padrões inéditos a respeito da relação entre o uso do espaço e de recursos alimentares de algumas espécies. Meus resultados sugerem que o marsupial $C$. lanatus ingere uma proporção de material de origem animal maior do que o relatado por outras metodologias. Além disso, o consumo de recursos com sinal $\mathrm{C} 4$ em mata de galeria por $O$. nigripes e $O$. bicolor indicam o uso de ambientes dominados por gramíneas C4 (campos adjacentes, clareiras ou bordas da mata de galeria). Por fim, os roedores em mata de galeria apresentaram amplitude e sobreposição de nicho isotópico maiores que em cerradão, e uma tendência a um padrão inverso foi observada para os marsupiais. Isso evidencia, por parte dos roedores, o consumo de uma diversidade maior de recursos na fitofisionomia mais complexa. Esse padrão sugere que esse grupo apresente maior plasticidade no uso de recursos alimentares que os marsupiais (Landry 1970) e que a disponibilidade e diversidade de recursos no ambiente provavelmente influencia a utilização de recursos e consequentemente a assimilação isotópica por essas espécies (Meserve et al. 1988). 


\section{Referências bibliográficas}

August, P. V. 1983. The role of habitat complexity and heterogeneity in structuring tropical mammal communities. Ecology 64:1495-1507.

Baugh, A. T., A. G. West, E. A. Rickart, T. E. Cerling, J. R. Ehleringer and M. D. Dearing. 2004. Stable isotope ratios ( $\delta 15 \mathrm{~N}$ and $\delta 13 \mathrm{C}$ ) of syntopic shrews (Sorex). The Southwestern Naturalist 49:493-500.

Bearhop, S., C. E. Adams, S. Waldron, R. A. Fuller and H. Macleod. 2004. Determining trophic niche width: a novel approach using stable isotope analysis. Journal of Animal Ecology 73:1007-1012.

Ben-David, M. and E. A. Flaherty [online]. 2012. Stable isotopes in mammalian research: a beginner's guide. Journal of Mammalogy 93:312-328.

Bocchiglieri, A., A. F. Mendonça and J. B. Campo. 2010. Diet composition of Gracilinanus agilis (Didelphimorphia , Didelphidae) in dry woodland areas of Cerrado in central Brazil. Mammalia 74:225-227.

Bonaventura, S. M. and O. V Suárez. 2001. Habitat use and diet in sympatric species of rodents of the low Paraná delta, Argentina. Mammalia 65:167-176.

Brown, J. H. 1975. Geographical ecology of desert rodents. Pp. 315-341 in Ecology and Evolution of Communities (M. L. Cody \& J. M. Diamond, eds.). Harvard University Press, Cambrigde.

Brown, J. H. and G. A. Lieberman. 1973. Resource utilization and coexistence of seed-eating desert rodents in sand dune habitats. Ecology 54:788-797.

Bustamante, M. M. C., L. A. Martinelli, D. A. Silva, P. B. Camargo, C. A. Klink, T. F. Domingues, et al. 2004. $15 \mathrm{~N}$ natural abundance in woody plants and soils of Central Brazilian Savannas (Cerrado). Ecological Applications 14:200-213.

Cáceres, N. C. 2002. Food habits and seed dispersal by the white-eared opossum, Didelphis albiventris, in Southern Brazil. Studies on Neotropical Fauna and Environment 37:97-104.

Cáceres, N. C. 2004. Diet of three didelphid marsupials (Mammalia, Didelphimorphia) in southern Brazil. Mammalian Biology 69:430-433.

Camargo, N. F. de. 2015. Uso dos estratos verticais por pequenos mamíferos em formações florestais do Cerrado brasileiro: padrões de diversidade, relação com a disponibilidade de recursos, seleção de hábitat e habilidade de locomoção arborícola das espécies. Universidade de Brasília.

Camargo, N. F. De, R. M. S. Cruz, J. F. Ribeiro and E. M. Vieira. 2011. Frugivoria e potencial dispersão de sementes pelo marsupial Gracilinanus agilis ( Didelphidae : Didelphimorphia ) em áreas de Cerrado no Brasil central Introdução. Acta Botanica Brasilica 25:646-656.

Camargo, N. F., J. F. Ribeiro, A. J. A. de Camargo and E. M. Vieira [online]. 2013. Diet of the gracile mouse opossum Gracilinanus agilis (Didelphimorphia: Didelphidae) in a neotropical savanna: intraspecific variation and resource selection. Acta Theriologica 59:183-191. 
Camargo, N. F., N. Sano and E. M. Vieira. no date. Predation upon small mammals by Caluromys lanatus (didelphimorphia: Didelphidae) and Callithrix penicillata (primates: callitrichidae) in the Brazilian savanna. Mammalia.

Campos, C., R. Ojeda, S. Monge and M. Dacar. 2001. Utilization of food resources by small and medium-sized mammals in the Monte Desert biome, Argentina. Austral Ecology 26:142-149.

Casella, J. and N. C. Cáceres. 2006. Diet of four small mammal species from Atlantic forest patches in South Brazil. Neotropical Biology and Conservation 1:5-11.

Codron, J., K. J. Duffy, N. L. Avenant, M. Sponheimer, J. Leichliter, O. Paine, et al. 2015. Stable isotope evidence for trophic niche partitioning in a South African savanna rodent community. Current Zoology.

Cody, M. L. 1968. On the methods of resource division in grassland bird communities. The American Naturalist 102:107-147.

Cody, M. L. and J. M. Diamond. 1975. Ecology and evolution of communities. Harvard University Press.

Crawford, K., R. A. Mcdonald and S. Bearhop. 2008. Applications of stable isotope techniques to the ecology of mammals. Mammal Review 38:87-107.

Cryan, P. M., C. A. Stricker and M. B. Wunder [online]. 2012. Evidence of cryptic individual specialization in an opportunistic insectivorous bat. Journal of Mammalogy 93:381-389.

Dalmagro, A. D. and E. M. Vieira. 2005. Patterns of habitat utilization of small rodents in an area of Araucaria forest in Southern Brazil. Austral Ecology 30:353-362.

DeNiro, M. J. and S. Epstein. 1978. Influence of diet on the distribution of carbon isotopes in animals. Geochimica et Cosmochimica Acta 42:495-506.

DeNiro, M. J. and S. Epstein. 1981. Influence of diet on the distribution of nitrogen isotopes in animals. Geochimica et Cosmochimica Acta 45:341-351.

Ellis, B. A., J. N. Mills, G. E. Glass, K. T. Mckee, D. A. Enria and J. E. Childs. 1998. Dietary habits of the common rodents in an agroecosystem in Argentina. Journal of Mammalogy 79:12031220.

Elton, C. 1927. Animal Ecology. The MacMillan Company, New York, NY.

Emmons, L. H. 1980. Ecology and resource partitioning among nine species of African rain forest squirrels. Ecological Monographs 50:31-54.

Ferreira, A. V., E. M. Bruna and H. L. Vasconcelos. 2011. Seed predators limit plant recruitment in Neotropical savannas. Oikos 120:1013-1022.

Fonseca, G. A. B. da and M. C. M. Kierulff. 1988. Biology and natural history of Brazilian Atlantic forest small mammals. Bulletin of the Florida State Museum, Biological Sciences 34:99-152.

Fry, B. 2008. Stable Isotope Ecology. P. 308pp in. Springer Science+Business Media, LLC, New York. 
Galetti, M., R. R. Rodarte, C. L. Neves, M. Moreira and R. Costa-Pereira [online]. 2016. Trophic Niche Differentiation in Rodents and Marsupials Revealed by Stable Isotopes. PloS one $11: \mathrm{e} 0152494$.

Giller, P. S. 1984. Community structure and the niche. Chapman and Hall, New York, NY.

Grelle, C. E. V. 2003. Forest structure and vertical stratification of small mammals in a secondary Atlantic Forest, southeastern Brazil. Studies on Neotropical Fauna and Environment 38:8185.

Grinnel, J. 1917. The niche-relationships of the California Thrasher. The Auk 34:427-433.

Hannibal, W. and N. C. Cáceres. 2010. Use of vertical space by small mammals in gallery forest and woodland savannah in south-western Brazil. Mammalia 74:247-255.

Hoeinghaus, D. J. and S. C. Zeug. 2008. Can stable isotope ratios provide for community-wide measures of trophic structure? Comment. Ecology 89:2353-2357.

Högberg, P. and I. J. Alexander. 1995. Roles of root symbioses in African woodland and forest: evidence from 15N abundance and foliar analysis. Journal of Ecology 83:217-224.

Hutchinson, G. E. 1957. Concluding remarks: Cold Spring Harbor symposium. Quant Biiol 22:415427.

Hutchinson, G. E. 1978. An introduction to population ecology. P. 260 in. Yale University Press, New Haven.

IBRAM. 2014. Guia de Unidades de Conservação do Distrito Federal. Brasília, DF.

Jackson, A. L., R. Inger, A. C. Parnell and S. Bearhop [online]. 2011. Comparing isotopic niche widths among and within communities: SIBER - Stable Isotope Bayesian Ellipses in R. The Journal of animal ecology 80:595-602.

Kelly, J. F. 2000. Stable isotopes of carbon and nitrogen in the study of avian and mammalian trophic ecology. Canadian Journal of Zoology 27:1-27.

Klink, C. A. and R. B. Machado. 2005. Conservation of the brazilian Cerrado. Conservation Biology 19:707-713.

Klopfer, P. H. and R. H. MacArthur. 1960. Niche size and faunal diversity. The American Naturalist 94:293-300.

Lajtha, K. and R. H. Michener. 1994. Stable isotopes in ecology and environmental science. Blackwell Scientific, London.

Landry, S. O. 1970. The rodentia as omnivores. The Quarterly Review of Biology 45:351-372.

Layman, C. A., M. S. Araujo, R. Boucek, C. M. Hammerschlag-Peyer, E. Harrison, Z. R. Jud, et al. [online]. 2012. Applying stable isotopes to examine food-web structure: an overview of analytical tools. Biological reviews of the Cambridge Philosophical Society 87:545-62. 
Layman, C. A., D. A. Arrington, C. G. Montaña and D. M. Post. 2007. Can stable isotope ratios provide for community-wide measures of trophic structure? Ecology 88:42-48.

Layman, C. A. and D. M. Post. 2008. Can stable isotope ratios provide for community-wide measures of trophic structure? Reply. Ecology 89:2358-2359.

Leiner, N. O., C. R. Dickman and W. R. Silva. 2010. Multiscale habitat selection by slender opossums (Marmosops spp.) in the Atlantic forest of Brazil. Journal of Mammalogy 91:561565.

Leite, Y. L. R., L. P. Costa and J. R. Stallings [online]. 1996. Diet and vertical space use of three sympatric opossums in a Brazilian Atlantic forest reserve. Journal of Tropical Ecology 12:435.

Lessa, L. G. and F. N. da Costa [online]. 2008. Diet and seed dispersal by five marsupials (Didelphimorphia: Didelphidae) in a Brazilian cerrado reserve. Mammalian Biology 75:10-16. Elsevier.

MacArthur, R. H. and J. W. MacArthur. 1961. On bird species diversity. Ecology 42:594-598.

MacArthur, R. and R. Levins. 1967. The limiting similarity, convergence and divergence of coexisting species. The American Naturalist 101:377-385.

Machado, R. B., M. B. R. Neto, P. G. P. Pereira, E. F. Caldas, D. A. Gonçalves, N. S. Santos, et al. 2004. Estimativas de perda da área do Cerrado brasileiro. Brasília, DF.

Mares, M. a., K. a. Ernest and D. D. Gettinger [online]. 1986. Small mammal community structure and composition in the Cerrado Province of central Brazil. Journal of Tropical Ecology 2:289300.

Martinelli, L. A., M. C. Piccolo, A. R. Townsend, P. M. Vitousek, E. Cuevas, W. McDowell, et al. 1999. Nitrogen stable isotopic composition of leaves and soil: Tropical versus temperate forests. Biogeochemistry 46:45-65.

Martins, E. G. and V. Bonato. 2004. On the diet of Gracilinanus microtarsus (Marsupialia , Didelphidae) in an Atlantic rainforest fragment in southeastern Brazil. Mammalian Biology 69:58-60.

Melo, C. de, A. M. da Silva and P. E. Oliveira. 2013. Oferta de frutos por espécies zoocóricas de sub-bosque em gradiente florestal do Cerrado. Biosciences Journal 29:2030-2041.

Melo, L. A. S., A. N. Moreira and F. de Silva. 2001. Armadilha para monitoramento de insetos.

Meserve, P. L. 1981. Resource partitioning in a Chilean semi-arid small mammal community. Journal of Animal Ecology 50:745-757.

Meserve, P. L., B. K. Lang and B. D. Patterson. 1988. Trophic relationships of small mammals in a Chilean temperate rainforest. Journal of Mammalogy 69:721-730.

Miranda, A. C., H. S. Miranda, I. de F. O. Dias and B. F. de S. Dias [online]. 1993. Soil and air temperatures during prescribed cerrado fires in Central Brazil. Journal of Tropical Ecology 9:313-320. 
Mittermeier, R. A., N. Myers, J. B. Thomsen, G. A. B. da Fonseca and S. Olivieri [online]. 1998. Biodiversity hotspots and major tropical wilderness areas: approaches to setting conservation priorities. Conservation Biology 12:516-520.

Mittermeier, R. A., W. R. Turner, F. W. Larsen, T. M. Brooks and C. Gascon [online]. 2011. Global biodiversity conservation: the critical role of hotspots. Pp. 3-22 in Biodiversity Hotspots (F. E. Zachos \& J. C. Habel, eds.). Springer Berlin Heidelberg, Berlin, Heidelberg.

MMA and IBAMA. 2011. Monitoramento do desmatamento nos biomas brasileiros por satélite, acordo de cooperação técnica MMA/IBAMA: Monitoramento do bioma Cerrado (2009-2010).

Newsome, S. D., C. Martínez del Rio, S. Bearhop and D. L. Phillips. 2007. A niche for isotopic ecology. Front Ecol Environ 5:429-436.

Newsome, S. D., M. T. Tinker, D. H. Monson, O. R. Oftedal, K. Ralls, M. M. Staedler, et al. 2009. Using stable isotopes to investigate individual diet specialization in California sea otters ( Enhydra lutris nereis ). Ecology 90:961-974.

Newsome, S. D., J. D. Yeakel, P. V Wheatley and M. T. Tinker [online]. 2012. Tools for quantifying isotopic niche space and dietary variation at the individual and population level. Journal of Mammalogy 93:329-341.

Nowak, R. M. 2005. Walker's marsupials of the world. P. 226 in. The Johns Hopkins University Press, Baltimore.

Oliveira-Filho, A. T. and J. A. Ratter. 2002. Vegetation physiognomies and woody flora of the Cerrado biome. P. 373p. in The Cerrados of Brazil: Ecology and natural history of a Neotropical savanna (P. S. Oliveira \& R. J. Marquis, eds.). Columbia University Press, New York, NY.

Paglia, A. P., A. B. Rylands, G. Herrmann, L. M. S. Aguiar, A. G. Chiarello, Y. L. R. Leite, et al. 2012. Lista Anotada dos Mamíferos do Brasil. P. 76pp in.

Parnell, A. C., R. Inger, S. Bearhop and A. L. Jackson [online]. 2010. Source partitioning using stable isotopes: coping with too much variation. PloS one 5:e9672.

Phillips, D. L. [online]. 2012. Converting isotope values to diet composition: the use of mixing models. Journal of Mammalogy 93:342-352.

Phillips, D. L. and J. W. Gregg [online]. 2003. Source partitioning using stable isotopes: coping with too many sources. Oecologia 136:261-9.

Pianka, E. R. 1973. The structure of lizard communities. Annual Review of Ecology and Systematics 4:53-74.

Post, D. M. 2002. Using stable isotopes to estimate trophic position: models, methods and assumptions. Ecology 83:703-718.

Ratter, J. A., J. F. Ribeiro and S. Bridgewater. 1997. The Brazilian Cerrado vegetation and threats to its biodiversity. Annals of Botany $80: 223-230$. 
Ribeiro, J. F. 2015. Uso de hábitat em diferentes escalas, distribuição da diversidade e nicho isotópico de comunidades de pequenos mamíferos do Cerrado central Juliana Fernandes Ribeiro. Universidade de Brasilia.

Ribeiro, J. F. and B. M. T. Walter. 2008. Fitofisionomias do Cerrado. Pp. 87-166 in Cerrado: Ecologia e Flora (S. M. Sano \& S. P. Almeida, eds.). EMBRAPA-CPAC, Planaltina-DF.

Rizzini, C. T. 1997. Tratado de fitogeografia do Brasil. $2^{a}$ edition. Âmbito Cultural Edições Ltda, Rio de Janeiro.

Rodríguez, M. a M. and L. M. Gerardo Herrera [online]. 2013. Isotopic niche mirrors trophic niche in a vertebrate island invader. Oecologia 171:537-44.

Rosenzweig, M. L. and J. Winakur. 1969. Population ecology of desert rodent communities: habitats and environmental complexity. Ecology 50:558-572.

Samuels, J. X. 2009. Cranial morphology and dietary habits of rodents. Zoological Journal of the Linnean Society 156:864-888.

Santori, R. T., L. G. Lessa and D. Astúa. 2012. Alimentação, nutrição e adaptações alimentares de marsupiais brasileiros. Pp. 385-406 in Os marsupais do Brasil: biologia, ecologia e conservação (N. C. Cáceres, ed.). $2^{\text {a }}$ edition. Editora UFMS, Campo Grande - MS.

Schmidt, S. and G. R. Stewart [online]. 2003. Delta15N values of tropical savanna and monsoon forest species reflect root specialisations and soil nitrogen status. Oecologia 134:569-77.

Schoener, T. W. 1974. Resource Partitioning in Ecological Communities. Science 185:27-39.

Semmens, B. X., E. J. Ward, J. W. Moore and C. T. Darimont [online]. 2009. Quantifying inter- and intra-population niche variability using hierarchical bayesian stable isotope mixing models. PloS one 4:e6187.

Simberloff, D. and T. Dayan. 1991. The guild concept and the structure of ecological communities. Annual Review of Ecology and Systematics 22:115-143.

Stallings, J. R. 1989. Small mammals inventories in an eastern Brazilian park. Bulletin of the Florida State Museum, Biological Sciences 34:153-200.

Sushma, H. S. and M. Singh [online]. 2006. Resource partitioning and interspecific interactions among sympatric rain forest arboreal mammals of the Western Ghats, India. Behavioral Ecology 17:479-490.

Symes, C. T., J. W. Wilson, S. M. Woodborne, Z. S. Shaikh and M. Scantlebury [online]. 2013. Resource partitioning of sympatric small mammals in an African forest-grassland vegetation mosaic. Austral Ecology 38:721-729.

Talamoni, S. A., D. Couto, D. A. Cordeiro Júnior and F. M. Diniz [online]. 2008. Diet of some species of Neotropical small mammals. Mammalian Biology 73:337-341.

Tieszen, L. L., T. W. Boutton, K. G. Tesdahl and N. A. Slade. 1983. Fractionation and turnover of stable carbon isotopes in animal tissues: Implications for delta13C analysis of diet. Oecologia 57:32-37. 
Tilman, D. 1982. Resource competition and community structure. Princeton University Press, Princeton, NJ.

Unesco. 2003. Subsídios ao zoneamento da APA Gama-Cabeça de Veado e Reserva da Biosfera do Cerrado : caracterização e conflitos socioambientais. P. 176pp in. UNESCO, MAB, Reserva da Biosfera do Cerrado, Brasília, DF.

Vanessa do Nascimento Ramos. 2007. Ecologia alimentar de pequenos mamíferos de áreas de cerrado no sudeste do Brasil. Universidade Federal de Uberlândia.

Vieira, E. M. and N. F. Camargo. 2012. Uso do espaço vertical por marsupiais brasileiros. Pp. 347364 in Os marsupais do Brasil: biologia, ecologia e conservação (N. C. Cáceres \& E. L. A. Monteiro-Filho, eds.). Editora UFMS, Campo Grande - MS.

Vieira, E. M. and J. Marinho-Filho. 1998. Pre- and post-fire habitat utilization by rodents of Cerrado from central Brazil. Biotropica 30:491-496.

Vieira, E. M. and D. A. de Moraes. 2003. Carnivory and insectivory in Neotropical marsupials. Pp. 271-284 in Predators with pouches: the biology of carnivorous marsupials (M. Jones, C. Dickman \& M. Archer, eds.). CSIRO Publishing.

Walker, B. H., G. Goldstein, G. Sarmiento, E. Medina, R. W. Ruess, P. G. H. Frost, et al. 1987. Determinants of tropical savannas. P. 156pp in. IRL Press Limited, Oxford, UK.

Wilman, H., J. Belmaker, J. Simpson, C. de la Rosa, M. M. Rivadeneira and W. Jetz. 2014. EltonTraits 1.0: Species-level foraging attributes of the world's birds and mammals. Ecology 95:2027. 


\section{Material suplementar}

Tabela suplementar 1. Número de indivíduos de espécies de pequenos mamíferos capturados em cerradão e em mata de galeria e número de amostras de pelos enviadas para análise de isótopos estáveis de $\delta^{13} \mathrm{C}$ e $\delta^{15} \mathrm{~N}$

\begin{tabular}{|c|c|c|}
\hline & № indivíduos & № amostras \\
\hline \multicolumn{3}{|l|}{ Caluromys lanatus } \\
\hline Cerradão & 4 & 4 \\
\hline Mata de Galeria & - & - \\
\hline \multicolumn{3}{|l|}{ Didelphis albiventris } \\
\hline Cerradão & 29 & 22 \\
\hline Mata de Galeria & 5 & 5 \\
\hline \multicolumn{3}{|l|}{ Gracilinanus agilis } \\
\hline Cerradão & 97 & 16 \\
\hline Mata de Galeria & 54 & 31 \\
\hline \multicolumn{3}{|l|}{ Cerradomys scotti } \\
\hline Cerradão & 1 & 1 \\
\hline Mata de Galeria & - & - \\
\hline \multicolumn{3}{|l|}{ Hylaeamys megacephalus } \\
\hline Cerradão & 11 & 8 \\
\hline Mata de Galeria & 10 & 9 \\
\hline \multicolumn{3}{|l|}{ Oecomys bicolor } \\
\hline Cerradão & 66 & 17 \\
\hline Mata de Galeria & 54 & 18 \\
\hline \multicolumn{3}{|l|}{ Rhipidomys macrurus } \\
\hline Cerradão & 6 & 4 \\
\hline Mata de Galeria & 38 & 14 \\
\hline \multicolumn{3}{|l|}{ Oligoryzomys nigripes } \\
\hline Cerradão & 4 & 2 \\
\hline Mata de Galeria & 28 & 12 \\
\hline \multicolumn{3}{|l|}{ Proechimys roberti } \\
\hline Cerradão & - & - \\
\hline Mata de Galeria & 6 & 4 \\
\hline
\end{tabular}


Tabela suplementar 2. Métricas de nicho isotópico (Layman et al. 2007) para comunidades de pequenos mamíferos em cerradão e mata de galeria calculadas a partir das médias dos valores de todas as espécies capturadas (todas as espécies) e somente as espécies que ocorreram em ambas fitofisionomias (espécies em comum). Espécies em cerradão: Caluromys lanatus, Didelphis albiventris, Gracilinanus agilis, Cerradomys scotti, Hylaeamys megacephalus, Oecomys bicolor, Rhipidomys macrurus e Oligoryzomys nigripes. Espécies em mata de galeria: Didelphis albiventris, Gracilinanus agilis, Hylaeamys megacephalus, Oecomys bicolor, Rhipidomys macrurus, Oligoryzomys nigripes e Proechimys roberti.

\begin{tabular}{lcccc}
\hline & \multicolumn{2}{c}{ Todas as espécies } & \multicolumn{2}{c}{ Espécies em comum } \\
& Cerradão & Mata de Galeria & Cerradão & Mata de Galeria \\
\hline TA & 22.724 & 6.299 & 17.774 & 6.299 \\
$\delta^{15} \mathrm{~N}$ Range & 4.618 & 3.318 & 4.618 & 3.318 \\
$\delta^{13} \mathrm{C}$ Range & 9.832 & 3.940 & 7.682 & 3.940 \\
CD & 3.407 & 1.548 & 2.764 & 1.617 \\
NND & 1.418 & 0.929 & 2.096 & 1.165 \\
SDNND & 0.925 & 0.580 & 2.526 & 0.376 \\
\hline
\end{tabular}


Tabela suplementar 3. Média e desvio padrão dos isótopos estáveis $\delta^{13} \mathrm{C}$ e $\delta^{15} \mathrm{~N}$ dos pelos de indivíduos de espécies de pequenos mamíferos capturados em áreas de cerradão e de mata de galeria do bioma Cerrado.

\begin{tabular}{|c|c|c|c|c|c|c|c|c|c|c|}
\hline \multirow{2}{*}{ Espécies } & \multicolumn{4}{|c|}{ Cerradão } & \multirow[b]{2}{*}{$\mathbf{n}$} & \multicolumn{4}{|c|}{ Mata de Galeria } & \multirow[b]{2}{*}{$\mathbf{n}$} \\
\hline & $\delta 13 C$ & $\delta 15 N$ & SDס13C & SDठ15N & & $\delta 13 C$ & $\delta 15 N$ & SDठ13C & SDठ15N & \\
\hline Caluromys lanatus & -21.89 & 5.16 & 0.24 & 0.83 & 4 & - & - & - & - & - \\
\hline Didelphis albiventris & -22.83 & 7.94 & 0.54 & 1.27 & 22 & -23.76 & 8.04 & 0.26 & 0.07 & 5 \\
\hline Gracilinanus agilis & -23.56 & 5.58 & 0.73 & 0.94 & 16 & -24.37 & 6.50 & 0.70 & 0.73 & 31 \\
\hline Cerradomys scotti & -14.29 & 4.06 & - & - & 1 & - & - & - & - & - \\
\hline Hylaeamys megacephalus & -24.11 & 3.51 & 0.37 & 0.80 & 8 & -25.04 & 5.89 & 1.56 & 0.82 & 9 \\
\hline Oecomys bicolor & -23.25 & 3.32 & 2.54 & 1.00 & 17 & -22.74 & 4.99 & 3.70 & 0.67 & 18 \\
\hline Oligoryzomys nigripes & -16.44 & 4.00 & 9.12 & 0.11 & 2 & -21.10 & 4.72 & 5.09 & 1.25 & 12 \\
\hline Proechimys roberti & - & - & - & - & - & -24.85 & 5.76 & 0.83 & 0.33 & 4 \\
\hline Rhipidomys macrurus & -24.12 & 3.39 & 0.29 & 0.83 & 4 & -23.65 & 4.80 & 0.71 & 0.80 & 14 \\
\hline
\end{tabular}


